\title{
Time reversibility and non deterministic behaviour in oscillatorily sheared suspensions of non-interacting particles at high Reynolds numbers
}

\begin{abstract}
Marcello Lappa
Department of Mechanical and Aerospace Engineering, University of Strathclyde, James Weir Building, 75 Montrose Street, Glasgow, G1 1XJ, UK - email: marcello.lappa@strath.ac.uk, marlappa@unina.it
\end{abstract}

ABSTRACT: Collections of inertial particles suspended in a viscous fluid and subjected to oscillatory shear have recently attracted much attention due to their relevance to a number of industrial applications and natural phenomena. It is known that, even at very low values of the flow Reynolds number, particle-to-particle interactions can lead to complex chaotic displacements despite the reversibility of the overarching fluid-dynamics (Stokes) equations. For high-Re flows, the loss of predictability after a finite time horizon is generically ascribed to the non-linear nature of the Navier-Stokes equations. Where the sources of nonlinearity are located exactly and how they influence the motion of particles, however, has not been clarified yet. We show that assuming particle interactions to be negligible, surprisingly, at high values of the Reynolds number the major source of non-deterministic behaviour comes from effects of stationary nature in the carrier flow. We report numerical simulations showing precisely how for geometries of finite extent such stationary effects emerge as the time-averaged non-linear response of the Navier-Stokes equations to the applied oscillatory forcing. They cause small deviations of the inertial particle's trajectory from the streamlines of the instantaneous oscillatory flow, which accumulate in time until the system behaviour becomes essentially non reversible.

\section{Nomenclature}

A cavity aspect ratio

d cavity thickness [m]

M Cycles of modulation

p Nondimensional pressure

$\widetilde{R} \quad$ solid particle radius [m]

Re Reynolds number

St Particle Stokes number

t Nondimensional time

$\mathrm{u} \quad$ Nondimensional velocity component along the $\mathrm{x}$ axis 
$\mathrm{V} \quad$ Nondimensional velocity

$\mathrm{V} \quad$ Nondimensional velocity component along the y axis

$\mathrm{x} \quad$ Nondimensional horizontal coordinate

y Nondimensional vertical coordinate

Greek symbols:

$\Lambda \quad$ Amplitude of wall oscillatory velocity [m/s]

$\Omega \quad$ Angular frequency [rad/s]

$v \quad$ Kinematic viscosity $\left[\mathrm{m}^{2} / \mathrm{s}\right]$

$\omega \quad$ Nondimensional angular frequency

$\delta \quad$ Nondimensional boundary layer thickness

$\psi \quad$ Nondimensional streamfunction

$\tau \quad$ Particle characteristic time

$\xi \quad$ Particle-to-fluid density ratio

Subscripts:

partcl particle

flow flow

L liquid

S solid

MAX maximum

\section{Introduction}

The field of particle dynamics is a large area encompassing a variety of phenomena with implications in several technological and industrial processes. A suspended phase in the form of particles is indeed widespread in a variety of fields.

As an example, the transport of an immiscible phase in a non-quiescent or convectively unstable (fluid) environment is relevant to research in powder metallurgy, catalytic processes, mineral processing and nuclear re-processing. In all these cases, involved materials pass through an initial liquid state (the so-called majority phase), in which a second immiscible phase (the minority phase) is present in the form of dispersed solid particles (see, e.g., Lappa, 2004 and 2005; Deng and Chawla, 2006). Such a concept is relevant to many inorganic materials with special features such as structural composites, many types of alloys, electrical conductors, ceramics and magnetic materials (Lee and Park, 1994; Ratke et al., 1996; Zhao et al.,1998; Zhang et al.,2006; Markov, 2008; Zhao et al., 2017).

It can even be applied to many organic substances produced in the context of protein crystallization research (Carotenuto et al., 2002; Lappa et al., 2003) and/or typical pharmaceutical processes for 
the production of medicines and drugs (e.g., the so-called continuous-cooling-crystallization-fromsolution-method, Brown et al., 2018). Indeed, such materials (proteins or other drugs) are obtained in the form of small crystals (seeds) nucleating from the liquid (typically a supersaturated phase). Existing (fundamental) lines of inquiry related to these subjects differ essentially in the mechanisms supporting particle dynamics, which, depending on their nature, can promote dispersion phenomena (leading to a more or less uniform distribution of particles inside the carrier liquid) or particle accumulation (formation of clusters).

Another distinction is obviously related to the type of suspension, which can be dilute or concentrated. In the latter case, attention must be paid to the nature and intensity of inter-particle forces. Particles can interact due to (attractive) electrostatic effects or hydrodynamic forces (which can be attractive or repulsive).

While electrostatic forces are, in general, dominant when particle are small and/or the value taken by the Reynolds number is close to zero, hydrodynamic (particle-particle) effects become increasingly more important as the size of particles and/or the Reynolds number grow. For such cases, however, also 'inertial' factors start to play a role. In practice, inertial effects are enabled every time a particle with finite size and mass is put in motion by a carrier flow.

Due to their spatial extension and non-negligible density difference with respect to the surrounding fluid, even if particle interactions or external body forces (such as gravity) are totally absent, inertial particles can experience convective disturbances that can cause a particle to depart from the trajectory that it would ideally follow if it had no mass and size. The most remarkable consequence of this process is that the velocity field associated with the motion of a set of particles is always non-solenoidal regardless of the nature (solenoidal or not) of the carrier flow. This peculiar property enables particles initially uniformly distributed in a fluid to produce concentration fluctuations (Benczik et al., 2002), eventually forming accumulation structures and/or depleted areas.

There is some consensus in the literature that, on increasing the Reynolds number, particletrajectory influential mechanisms operative at the particle scale are gradually transferred from the aforementioned particle-to-particle mutual interference to purely inertial processes like those described above. As these mechanisms do not rely on inter-particle effects, they can be effective even in dilute suspensions. In such cases, the nature (structure and topology) of the considered carrier flow becomes obviously an important aspect of the problem. The intrinsic level of vorticity possessed by the flow can indeed have a remarkable impact on the dynamics of the dispersed phase. Particles denser than the fluid are typically radially ejected towards vortex periphery due to centrifugal effects (where they accumulate forming aggregates with fractal topology) or captured in the vortex core in the opposite situation in which their density is smaller. Both effects can cause particle preferential concentration (Eaton and Fessler, 1994; Raju and Meiburg, 1995; Chen et al., 2006; Lappa, 2018). The situation becomes even more interesting if the considered flow is featured by the propagation of waves. Interesting lines of inquiry have been initiated over recent years about the ability of some thermally-driven natural flows (Schwabe et al., 1996) to promote the formation of well-defined particle structures when they are in unsteady (time-periodic) conditions (see also 
Sapsis and Haller, 2010). Regardless of the specific force driving fluid flow (be it buoyancy or surface-tension), very regular aggregates can be formed, the only necessary prerequisite being represented by the existence of a single or more waves travelling in the flow (Schwabe et al., 2007; Schwabe and Mizev, 2011; Pushkin et al, 2011; Lappa, 2014a; Melnikov et al., 2011; Kuhlmann et al., 2014; Gotoda et al., 2016). Rather than being the mere consequence of centrifugal effects, these phenomena are supported by a delicate synchronization process established between the vorticity associated with particles and the angular frequency of the just mentioned waves (Pushkin et al., 2011; Lappa, 2013abc).

Similar phenomena can be produced if particles are placed in a fluid undergoing thermovibrational convection (Shevtsova et al., 2010, 2011ab, Lappa, 2016c). Indeed, as revealed by these studies (Lappa, 2014b, 2016a, 2017a), by properly tuning the frequency of applied vibrations and their direction with respect to the applied temperature difference it is possible to drive particles to specific subregions of the considered container where they accumulate forming well-defined geometric objects resembling the perfection of the typical "quadrics" of projective geometry (open or compact surfaces such as cylinders, paraboloids, "ovoids” and conical surfaces). The essentially inertial nature of these phenomena, yet induced by a kind of synchronization between the inertial response of particles and the time-periodic behaviour of the carrier flow (Lappa, 2016b), distinguishes them from another category of vibrationally induced clustering mechanisms in concentrated systems, which rely essentially on inter-particle hydrodynamic forces (Tabakova and Zapruanov, 1982ab; Wunenburger et al., 2002; Ivanova et al., 2005; Kozlov et al., 2006).

In the present work, we address the companion problem in which the oscillatory nature of the carrier flow is not due to the presence of a time-varying acceleration in a non-isothermal fluid or the emergence of waves in the considered thermally-driven natural flows. Rather it follows from the application of a periodic shear through back and forth (harmonic) motion of the walls limiting the fluid domain from above and from below (a double lid-driven isothermal cavity).

Moreover, rather than assessing the ability of the flow to produce accumulation structures, we explore just the opposite conundrum that is its ability to retain the morphology and cohesive nature of a set of particles initially assumed to be concentrated in an area having circular shape (initial conditions corresponding to a particle-dense 'ball'; we will be more precise about the meaning of the adjective 'cohesive' later).

There is a long tradition of studies on this subject, essentially focusing on the role played by interparticle forces in the limit of very viscous flow (for which the Navier-Stokes equations become linear, and therefore any source of nonlinearity has to be related to particle interactions of various natures). In general, different contributions have to be considered: (i) the long-range hydrodynamic interactions, (ii) lubrication, which results from the thin layer of viscous fluid that separates nearly touching particles, (iii) contacts, which prevent particles from overlapping, (iv) surface asperities that can induce particle displacement, forcing particles from their reversible path.

In such a context, for instance, it is worth mentioning the very interesting line of inquiry originating from the experiments performed by Pine et al. (2005), who investigated the asymptotic behaviour of 
solid-liquid systems governed by the Stokes equations (collections of neutrally buoyant spheres suspended in a viscous fluid and subjected to a periodic shear in the limit where dissipation dominates and inertia is ignored). A remarkable phase transition separating reversible from irreversible dynamics was identified as the strain amplitude or volume fraction were increased (the reader being also referred to the later excellent works by Metzger and Butler, 2010 and 2012, and Metzger et al., 2013).

By virtue of these studies it is known that there is a concentration dependent threshold for the deformation or strain beyond which particles do not return to their starting configurations after one or more cycles (reversing the direction of flow does not in practice lead to a time-reversed motion for all of the particles, which rather tend to follow the statistics of an anisotropic random walk).

This class of experiments illustrated the potential irreversible nature of particulate systems submitted to an oscillatory shear in the limit of negligible inertia.

A proper assessment of the contribution brought to the loss of time reversibility (and system predictability) by other sources of non-linearity, however, is still missing. There is some general and diffused awareness in the literature that, in the absence of particle-particle interference and for high values of the Reynolds number, the non-linear nature of the Navier-stokes equation must play a role, but the precise cause-and-effect relationships supporting this assumption are still poorly understood. Also the role of particle inertia, potentially contributing to irreversibility via the accumulation/capturing mechanisms discussed before, is still unknown.

Resolving the questions requires separating the different contributions to the particle dynamics and estimating their relative importance. This is the reason why here we concentrate expressly on the high-Re case neglecting particle interactions and retroaction of the particles on the motion of the fluid and explore the role of inertial and non linear effects.

\section{Mathematical Model}

\subsection{The Geometry}

The specificity of the considered situation lies in the peculiar nature of the driving imposed velocity at the walls, which has zero-time averaged value and which, accordingly, makes linear effects negligible, thereby enabling the accumulation in time of nonlinear effects only.

For simplicity, we consider a cavity with characteristic depth d shown in Fig.1. The system aspect ratio (A), defined as its length-to-depth ratio ( $\ell / d$ ) is fixed to 4 as in the earlier studies by Lappa (2016c) about thermovibrational convection. The top and bottom horizontal walls are assumed to move in opposite directions with velocity $\pm \Lambda \sin (\Omega t$ ), respectively (a double lid-driven cavity), which make this system an ideal target to study the dynamics of sheared dispersions with shear reversal of varying strain amplitude in well-controllable conditions. 


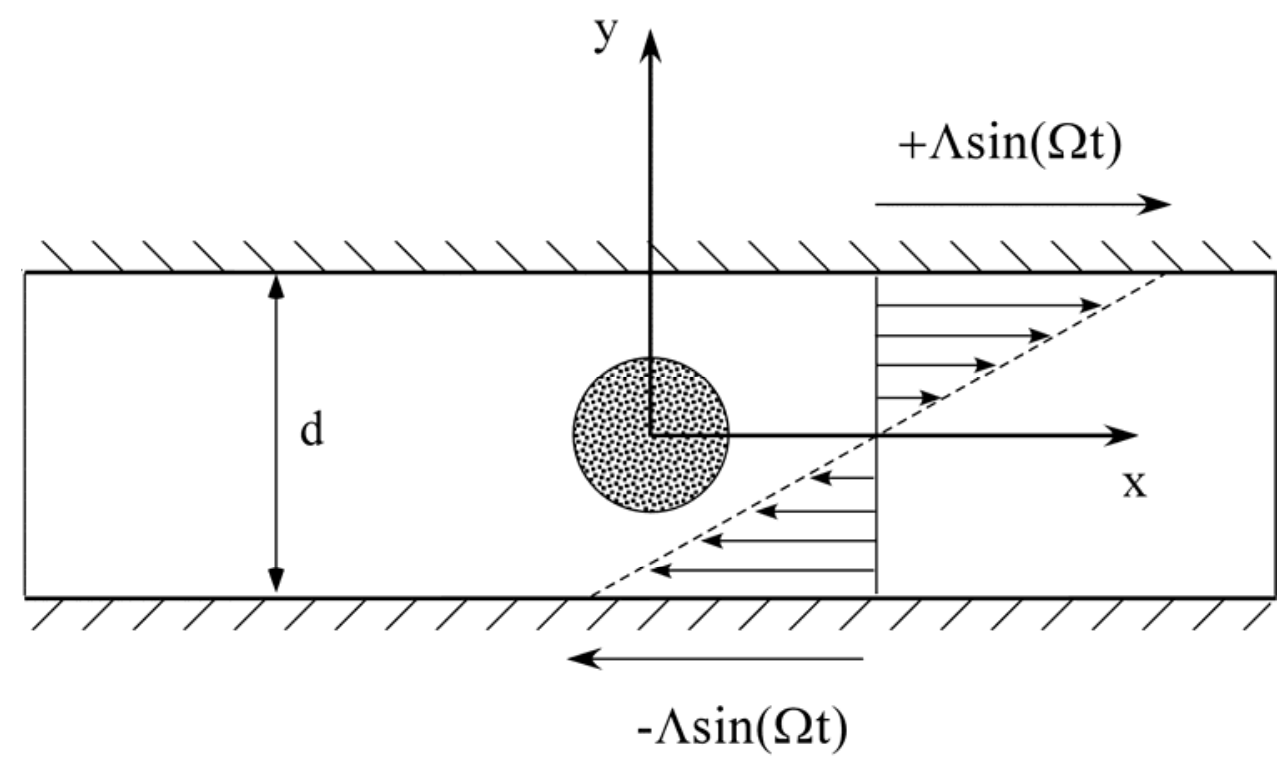

Figure 1: Sketch of the considered problem

\subsection{The governing equations for the fluid phase}

The fluid is assumed to be viscous and incompressible. Accordingly, introducing the substantial derivative as:

$\frac{D \underline{V}}{D t}=\frac{\partial \underline{V}}{\partial}+\underline{\nabla} \cdot[\underline{V} \underline{V}]$

the balance equations can be cast in compact non-dimensional form as:

$\underline{\nabla} \cdot \underline{V}=0$

and

$\frac{D \underline{V}}{D t}+\underline{\nabla} p=\nabla^{2} \underline{V}$

having assumed as reference quantities for the space coordinates, velocity, pressure and time, $d, v / d$, $\rho_{\mathrm{L}}(v / \mathrm{d})^{2}$ and $\mathrm{d}^{2} / v$, respectively (where $\rho_{\mathrm{L}}$ and $v$ are the fluid density and kinematic viscosity, respectively). Accordingly the non-dimensional shear frequency reads:

$\omega=\frac{\Omega d^{2}}{v}$

and the boundary condition at the moving walls can be re-written as: 
$V= \pm \operatorname{Re} \sin (\omega t)$ at $y= \pm 1 / 2$

where

$\operatorname{Re}=\frac{\Lambda d}{v}$

No slip conditions are assumed on the lateral non-moving walls.

\subsection{The Dispersed Phase}

The considered problem obviously requires a multi-scale approach given the intrinsic need to deal at the same time with large-scale (in our case shear-driven oscillatory flow) and small-scale phenomena (particles and their motion under the influence of large-scale flow). From a purely mathematical standpoint, this means that a modelling hierarchy must be defined and implemented to account for different mechanisms at different scales. Sifting through the existing literature about multiphase flows, one would realize that different strategies have been elaborated over the years to deal with such a challenging problem. However, selecting a specific technique often is not as straightforward as one would imagine as each approach is characterized by specific advantages and drawbacks. Here we base the selection process on some simple arguments and practical reasoning.

As our particles are assumed to be undeformable (spheres with constant diameter, i.e. a monodisperse distribution), there is no need to track their interface explicitly, i.e. by means of a front-tracking method. This would even not be practically feasible given the extremely high number of particles that we consider in order to get statistically meaningful information. Accordingly, we model all the particles as point entities with respect to convective structures by which they are transported. Since we are still interested in inertial effects, however, particles are assumed to possess finite mass and size, this being accounted for through the solution of a Lagrangian transport equation directly derived from Newton's second law (Lappa and Carotenuto, 2003) in which each force exerted by the fluid on the particle is modelled as a specific term. This means that a hybrid approach is used to deal with the present problem, to be considered as a 'Lagrangian' one in terms of particle transport and as an 'Eulerian' one for what concerns fluid-dynamic effects (eqs. (2) and (3)). The former is formally reduced to the integration in time of the so-called Maxey-Riley equation (Maxey and Riley, 1983; Michaelides, 1997; Lappa, 2013c), which in non-dimensional form can be cast in compact form as:

$$
\frac{d \underline{V}_{\text {partcl }}}{d t}=\frac{1}{\xi+1 / 2}\left[\frac{\left(\underline{V}-\underline{V}_{\text {partcl }}\right)}{S t}+\frac{3}{2} \frac{D \underline{V}}{D t}+\sqrt{\frac{9}{2 \pi} \frac{1}{S t}} \int_{o}^{t} \frac{1}{\sqrt{t-\tilde{t}}} \frac{d}{d \tilde{t}}\left(\underline{V}-\underline{V}_{\text {partcl }}\right) d \tilde{t}\right]
$$


where $\underline{V}_{\text {part }}$ and $\underline{V}$ are the particle and fluid velocity, respectively. Moreover, $\xi$ is the ratio of the particle to the fluid density $\left(\rho_{S} / \rho_{\mathrm{L}}\right)$ and $\mathrm{St}$ is the particle Stokes number. In particular, the terms in order of appearance on the right-hand side have the following physical meaning: the Stokes drag, the force exerted by the undisturbed flow on the particle and the Basset history force. This equation also takes into account the added mass effect resulting from part of the fluid moving with the particle (for some interesting historical details the reader may consider Coimbra and Rangel, 2001; Haller and Sapsis, 2008). Since in the present work, as outlined before, we explicitly decide to ignore inter-particle effects (as we target an improved understanding of non-linearities being brought in by the non-linear nature of the Eulerian facet of the problem), the equation does not account for particle hydrodynamic interactions, lubrication or contacts. For the same reasons, we do not consider the presence of gravity (potentially pulling particles down). Moreover, the coupling with the fluid is in one direction only (particles being influenced by the surrounding fluid, the vice versa not being considered, i.e. the so-called “one-way” model).

The underlying assumptions on which eq. (7) is based are a perfectly spherical shape of the solid particles and a very small value $(<<1)$ of the particle to fluid system characteristic size ratio $a=\tilde{R} / d$ (where $\widetilde{R}$ is the solid particle radius). Moreover, the so-called particle relaxation (response) time must be much less than the characteristic time scales of the considered flow (Michaelides, 1997).

The particle relaxation time $(\tau)$ can be defined as:

$\tau=\frac{2}{9} \frac{\widetilde{R}^{2}}{v}$

We introduce a very general characteristic flow time scale as:

$\tau_{\text {flow }}=\frac{d^{2}}{v}$

Other relevant time scales, however, must be considered taking into account the peculiarities of the specific flow considered (essentially its strength and unsteadiness). Indeed, the dynamics involves two scales which are determined by $\Omega$ and $\Lambda$ and there is a priori no reason why they should be of the same order of magnitude These time scales read:

$\tau_{\omega}=\frac{2 \pi}{\Omega}$

and

$\tau_{\Lambda}=\frac{d}{\Lambda}$ 
Non-dimensional parameters accounting for the relative importance of the particle relaxation time and these characteristic times, in turn, can be naturally defined (see Hjelmfelt and Mockros, 1966; Coimbra and Rangel, 2001) as :

$$
\begin{aligned}
& S t=\tau \frac{v}{d^{2}}=\frac{2}{9} a^{2} \\
& S t_{\Omega}=\tau \frac{\Omega}{2 \pi}=\frac{a^{2}}{9 \pi} \omega=\frac{1}{2 \pi}(S t) \omega \\
& S t_{\Lambda}=\tau \frac{\Lambda}{d}=\frac{2}{9} a^{2} \operatorname{Re}=(S t) \operatorname{Re}
\end{aligned}
$$

The first appears expressly in eq. (7). The second (eq. (10b)) also deserves some specific attention given its role in determining the ability of particles to attain a synchronous behaviour with respect to the background oscillatory velocity field.

Indeed, as illustrated by Simic-Stefani et al. (2006), the limit $\mathrm{St}_{\Omega} \rightarrow 0$ will represent the condition for which a fixed viewer would see particles 'frozen' in the flow field, the opposite limit $\mathrm{St}_{\omega}>>1$, representing situations in which particle would lose the capability to follow any velocity change in the flow.

To make eq. (7) valid, all these dimensionless parameters (St, $\mathrm{St}_{\Omega}$ and $\mathrm{St}_{\Lambda}$ ) must be sufficiently small $(<<1)$. The following additional conditions must therefore be satisfied:

$\mathrm{St}<<1$

$\omega<<2 \pi / \mathrm{St}$

$\operatorname{Re}<<\mathrm{St}^{-1}$

As for the case considered in the present work we consider $\mathrm{St}=10^{-4}$, the validity of eq. (11a) is implicitly guaranteed. Equation (11b), however, puts a constraint on the maximum non-dimensional frequency, which for the considered value of St has to be $\omega<2 \pi \times 10^{4}$. Here, in particular, to be on a safe side the maximum $\omega$ is fixed to $\omega=10^{4}$. Another constraint restricting the range of admissible parameters comes from eq. (11c), which places a limit on the Reynolds number $\left(\operatorname{Re}<10^{4}\right)$.

In addition to the above restrictions, however, conditions must be also satisfied for which the socalled history term (the Basset force, given by the integral contribution in eq. (7)) can be neglected. The inclusion of this term in the model would make the particle equation non-explicit in the velocity or acceleration, leading to a cumbersome and computationally time-consuming numerical problem (intractable for the extensive number of particles considered in this work).

Although the knowledge of the motion of solid particles in sinusoidally varying velocity fields seems to be still rather limited for high-Re flows, some useful information on the Basset force can be found in the existing literature for the case of relatively simple velocity fields. As an example, 
interesting studies of particle dynamics under the effects of small sinusoidal fluctuations in the freestream velocity (i.e. harmonic background fluid velocity) are due to Lighthill (1954), Houghton (1961), Herringe (1977), Mei et al. (1991), Vojir and Michaelides (1994) and Coimbra and Rangel (2001). Other important investigations have dealt with the terminal velocity of a spherical particle in a vertically oscillating liquid (among them, it is worth mentioning Baird et al., 1967; Tunstall and Houghton, 1968 and Ikeda, 1989). For the case of particles undergoing a sinusoidal motion or subjected to vibrations in an otherwise quiescent fluid, we may cite Langbein (1991), Thomson et al. (1997) and Simic-Stefani et al. (2006).

In the following we refer to the general criterion provided by Hjemfelt and Mockros (1966) for turbulent flows, by which the effect of the Basset term should be considered negligible when the Stokes number (all variants) satisfies the condition $<2 / 9 \xi^{1 / 2} \cong 3 \times 10^{-1}$.

An evaluation of St, $\mathrm{St}_{\Omega}$ and $\mathrm{St}_{\Lambda}$ with the present choice of parameters (we assume $\xi=2$ ) shows that these criteria are met with $\mathrm{St}=10^{-4}, \mathrm{St}_{\Omega} \cong 1.6 \times 10^{-1}$ and $\mathrm{St}_{\Lambda} \cong 10^{-1}$ (assuming for $\omega$ and Re the worst cases, i.e. their largest considered values $\omega=10^{4}$ and $\mathrm{Re}=10^{3}$, respectively).

Accordingly, with the two-fold purpose to make the above requirements satisfied and explore a variety of dynamical regimes, we assume the following conditions for the numerical simulations: $\mathrm{A}=4, \xi=2, \mathrm{St}=10^{-4}$ and the following ranges of $\mathrm{Re}$ and $\omega, 0<\mathrm{Re} \leq 10^{3}$ and $10<\omega \leq 10^{4}$.

\section{The Numerical Method}

An extensive description of the numerical method used here to solve the incompressible NavierStokes equations can be found, e.g., in Lappa (1997, 2018). This technique can essentially be seen as a spin-off of the Ladyzhenskaya (1969) inverse theorem of calculus, by which every vector field can always be mathematically represented as the superposition of a field with given curl (representing the flow 'vorticity' when the considered unknown vector is the fluid velocity) and divergence (measuring the 'compressibility' of the considered fluid phenomena). Such a theorem may be regarded as the mathematical basis of a series of techniques known as projection methods, where the constraints represented by the need to make the velocity field solenoidal (zero divergence) and let it develop the correct amount vorticity, are enforced separately. The latter requirement is indeed implemented through the solution of a simplified ('intermediate') version of the momentum equation where the presence of the pressure terms is neglected (these terms having no impact on the curl of the velocity field). The former is taken into account via the solution of an additional equation derived by assuming the physical velocity field to be a linear combination of the intermediate field and the pressure gradient and forcing it into the continuity equation (the solution of this equation provides indeed the missing pressure field that is finally used to 'correct' the velocity).

In the present work, the above method has been implemented using an explicit-in-time approach with central differences and the QUICK scheme used for the discretization of the diffusive and convective terms, respectively. The particle transport equation has been integrated in time using a 
forth-order accurate Runge-Kutta scheme (Atkinson, 1989). The resulting algorithm has been already tested and validated in earlier works of the present author by considering the interplay of particles with different types of convection, namely, thermogravitational, thermocapillary and thermovibrational flows (Lappa, 2013abc,2014ab, 2016ab, 2017, 2018) and even related hybrid states.

\subsection{Time-averaged and instantaneous effects}

In the present work, the additional time-averaged Eulerian velocity field $\bar{V}(\bar{u}, \bar{v})$, not provided directly by the implementation of the projection method described before, has been determined in a post-computation stage as

$$
\underline{\bar{V}}=\frac{\omega}{2 \pi} \int_{0}^{2 \pi / \omega} \underline{V} d t
$$

with the related streamfunction $\bar{\psi}$ being obtained via solution of the equation:

$$
\nabla^{2} \bar{\psi}=\frac{\partial \bar{u}}{\partial y}-\frac{\partial \bar{v}}{\partial x}
$$

The significance of these quantities can readily be illustrated by noting that if the balance equations governing fluid motion (Eqs. (1-3)) were linear, there would be no time-averaged effects as the average value in time of the forcing affecting the considered problem is zero, i.e. :

$$
\frac{\omega}{2 \pi} \int_{0}^{2 \pi / \omega} \operatorname{Re} \sin (\omega t) d t=0 \text { at } y= \pm 1 / 2
$$

The time-averaged velocity field can therefore be regarded as a consequence of the non-linear nature of the Navier-Stokes equations. The velocity field provided by the solution of eqs. (1-3) can be expressed accordingly as $\underline{V}=\underline{\bar{V}}+\underline{V}^{\prime}$ where $\underline{V}$ is the fluctuating contribution emerging as a linear response to applied wall motion. The derived function $\bar{\psi}$ is also a useful quantity as one may argue that in the regions where $\bar{\psi} \cong 0$, the system will display approximately a linear behaviour (direct proportionality between the forcing and the fluid velocity) with the non-linear effects being spatially localized in the regions where $\bar{\psi}$ attains relatively high values (which explains why in the following we will pay special attention to the patterning behavior of this function). 


\subsection{The Initial Conditions}

We consider an initial distribution of $\mathrm{N}_{\mathrm{r}} \mathrm{x} \mathrm{N}_{\varphi}=5 \times 10^{3}$ spherical particles uniformly distributed over a circular region of non-dimensional radius $r=0.3$ (hereafter simply referred to collectively as the 'ball' of initial conditions, with 50 particles along the radial direction and 100 particles along the azimuthal direction at any radial position).

To filter out transient effects, such ball is introduced in the computation at the time $\mathrm{t}=\mathrm{t}^{*}$ at which the time-averaged fluid velocity defined by eq. (12) has reached steady conditions, i.e. $\partial \underline{\bar{V}} / \partial t=0$ (starting from this instant, time is redefined as $\bar{t}=t-t *$ so to have $\bar{t}=0$ as the initial time).

The key idea is to perform successive cycles of shear starting from $\bar{t}=0$ over a given timeframe and measure the evolution of the shape of the cloud of particles at the end of each cycle (this approach being particularly advantageous for investigating the transition between reversible and irreversible dynamics). In particular, in the following we will classify the dynamics in the considered timeframe as:

- Apparently stationary if particles do not display any appreciable (visible) motion.

- Reversible if particle trajectories starting from the initial cloud end on it anyway and the cloud recovers periodically its initial spherical shape

- Partially irreversible (or borderline) if the cloud of particles undergoes changes in shape as the number of performed cycles increases but the set of particles remains a cohesive entity for long times, maintaining a sharp boundary between its particle-filled interior and the clear fluid outside.

- Irreversible if the motion of the particles is no longer deterministic and the ball is destroyed with the ensuing onset of completely chaotic dynamics.

\subsection{Validation}

The present algorithm for the solution of the Navier Stokes in the presence of moving top and bottom walls was validated through comparison with analogous results available in the literature (well-defined benchmarks). In particular we have considered the simulations performed for the classical two-sided lid-driven square cavity (case with antiparallel wall motion and constant imposed velocity) by Perumal and Dass (2008) and Karmakar and Pandit (2015) and a representative value of the Reynolds number $\left(\mathrm{Re}=10^{3}\right.$, see Figs. 2-3). 


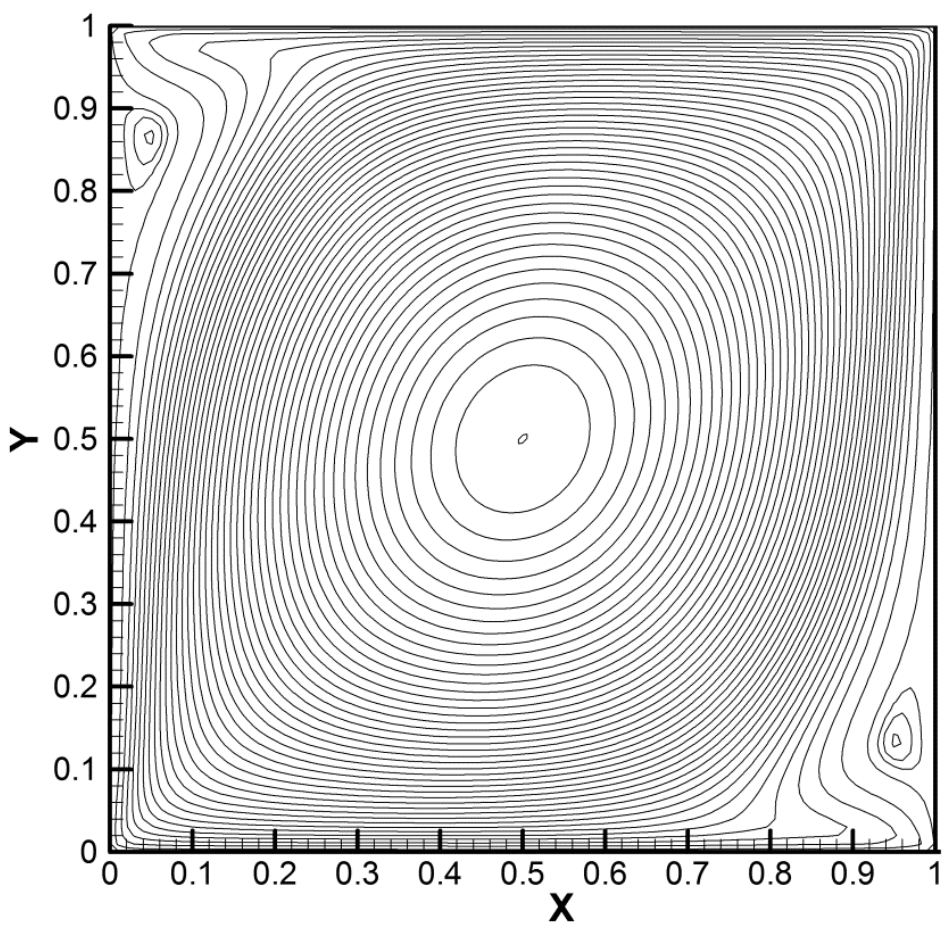

Figure 2: Streamlines for the classical two-sided lid-driven square cavity problem $\left(A=1, \operatorname{Re}=10^{3}\right.$ : mesh 100x100, $\left.\psi_{\max }=155.5\right)$.

Table I: Position of the primary and secondary vortices for the two-sided lid-driven square cavity and $\mathrm{Re}=10^{3}$ : present mesh 100x100; ${ }^{*}$ FDM results after Perumal and Dass (2008) (Finite Difference Method with a 129x129 grid), " LBM results after Perumal and Dass (2008) (Lattice Boltzmann Method with a 257x257 grid), ${ }^{* *}$ Karmakar and Pandit (2015) (Finite Difference Method with compact 5-point stencil scheme).

\begin{tabular}{lllllll}
\hline \hline Source & $\mathrm{X}_{\text {centervortex }}$ & $\mathrm{y}_{\text {centervortex }}$ & $\mathrm{X}_{\text {rightvortex }}$ & $\mathrm{Y}_{\text {rightvortex }}$ & $\mathrm{X}_{\text {leftvortex }}$ & $\mathrm{Y}_{\text {leftvortex }}$ \\
\hline \hline $\mathrm{FDM}^{*}$ & 0.5007 & 0.4981 & 0.9507 & 0.1319 & 0.0492 & 0.8663 \\
LBM $^{*}$ & 0.5012 & 0.4982 & 0.9512 & 0.1326 & 0.0449 & 0.8609 \\
$\begin{array}{l}\text { Compact } \\
\text { Scheme }\end{array}$ & 0.5000 & 0.5000 & 0.9583 & 0.1333 & 0.0417 & 0.8667 \\
Present & 0.5002 & 0.4996 & 0.9503 & 0.1320 & 0.0449 & 0.8670 \\
\hline \hline
\end{tabular}

As shown in Fig. 3, the present velocity profiles obtained with grids 100x100 and 200x200 are almost indistinguishable. Moreover, they are in excellent agreement with the results that Perumal and Dass (2008) obtained using two completely different numerical approaches (namely a finite difference method with a 129x129 mesh and a lattice boltzmann method with a 257x257 grid). The very good agreement also holds for the positions of the main and minor rolls present in the system, as witnessed by the data reported in Table I (the reader is also referred to the pattern in Fig. 2). 


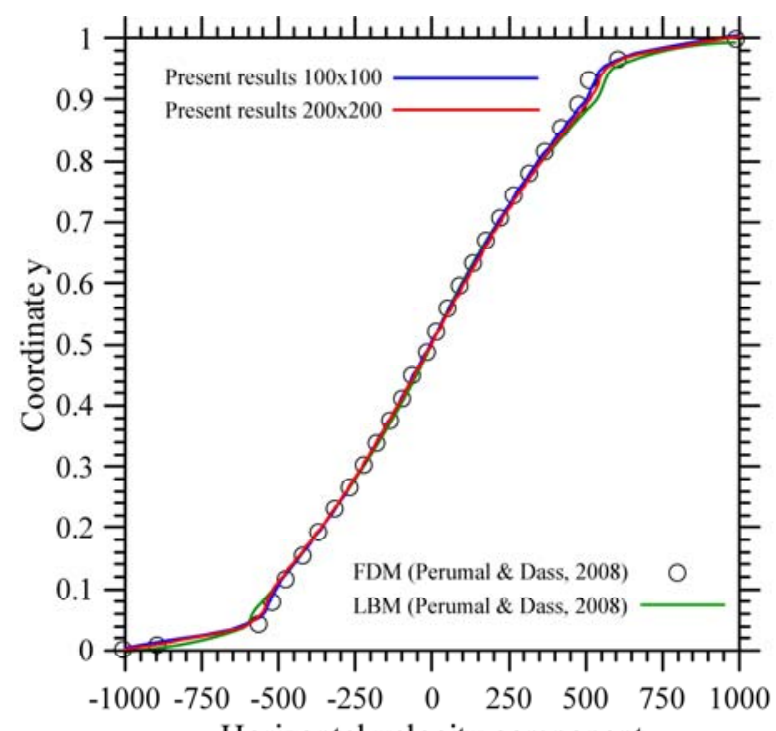

Horizontal velocity component

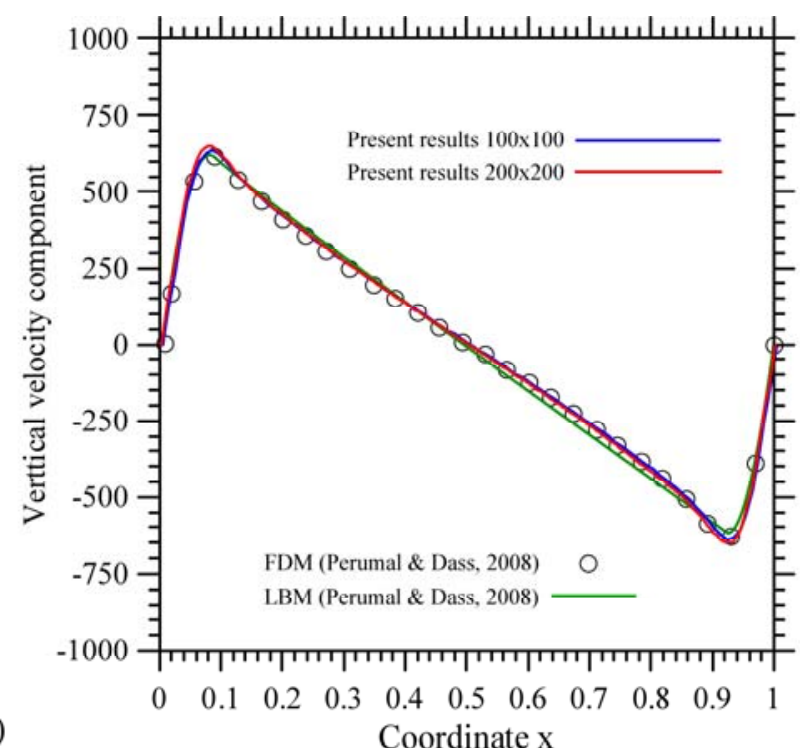

b)

Figure 3: Velocity profiles for the classical two-sided lid-driven square cavity $\left(\operatorname{Re}=10^{3}\right)$ : a) Horizontal velocity component as a function of $\mathrm{y}$, b) Vertical velocity component as a function of $\mathrm{x}$.

With regard to the assessment of the inertial particle tracking method against other data in the literature, relevant information can be found in Lappa (2013c). Indeed, the kernels (subroutines) used to track inertial particles in the present work are the same already used in earlier studies by the present author. Their reliability was tested considering the dynamics of particles in situations relevant to the cases considered here, namely, oscillatory flow, flow driven by imposed motion along the external boundaries, motion of particles dominated by inertia and no particle interactions. This has been achieved via direct comparison with the results by Melnikov et al. (2011) for the same values of the Stokes number and density ratios considered in the present study, i.e. $\mathrm{St}=10^{-4}$ and $\xi \cong 2$, and $R e \cong 2500$ (this information is not duplicated here as it can be found in Lappa, 2013c). The same subroutines were also used by Lappa (2014ab). Here we limit ourselves to recalling that solution of eq. (7) requires coupling between the CFD and particle tracking kernels, which in the aforementioned subroutines has been implemented on the basis of a linear interpolation approach (to determine the fluid velocity and related derivatives at the location of any trcked particle as a function of the corresponding values taken by $\underline{V}$ on the fixed nodes of the staggered grid).

\subsection{Grid Refinement Study}

The numerical approach has also been tested by checking its convergence under mesh refinement for the same conditions examined in Sect. $4(\mathrm{~A}=4$, antiparallel wall motion and imposed wall velocity varying sinusoidally in time).

The identification of a relevant (representative) case for the grid refinement study has been based on relevant arguments and correlations available in the literature. Relevant information along these lines can be found, e.g., in the earlier investigations by Ilin and Morgulis (2012 and 2015), where it 
has been shown that an oscillating flow of a viscous incompressible fluid between two solid walls produced by transverse vibrations of the walls generally produces boundary layers with nondimensional thickness scaling as $\delta \cong \omega^{-1 / 2}$. However, one has also to take into account that, according to the classical boundary layer theory, the thickness of the boundary layer is expected to be inversely proportional to the square root of the Reynolds number ( $\delta \cong \operatorname{Re}^{-1 / 2}$, Prandtl, 1938). With these propaedeutical considerations in mind, we have decided to test the convergence of the algorithm under the most restrictive (challenging from a purely numerical point of view) possible conditions, namely, the highest values of the nondimensional frequency considered in the present work and the highest value of the Reynolds number $\left(\omega=10^{3}\right.$ and $\left.\operatorname{Re}=10^{3}\right)$. For these conditions either the vibrational boundary layer or the classical viscous boundary layer (or eventually both) are expected to be present in the fluid domain (thinnest convective structures recognisable in the system).

A grid refinement study also requires proper definition of "sensitive quantities" for the evaluation of percentage variations to be used to judge on the convergence process. In this regard, we have considered both "local" and global "quantities", the former corresponding to the maximum value of the time-averaged velocity components along the $\mathrm{x}$ and $\mathrm{y}$ directions and the latter being the integrals of such quantities extended to the entire computational domain, namely:

$$
\begin{aligned}
& \widetilde{U}=\int_{\Omega}|\bar{u}| d \Omega \\
& \widetilde{V}=\int_{\Omega}|\bar{v}| d \Omega
\end{aligned}
$$

Table II: Maximum time averaged velocity components and related integral quantities as a function of the (uniform) mesh density $\left(\mathrm{A}=4, \mathrm{Re}=1000, \omega=1000, \widetilde{Q}^{+}\right.$and $\widetilde{Q}^{-}$evaluated for $\mathrm{t} \cong 0.03$ ).

\begin{tabular}{lllllll}
\hline \hline Mesh & $\bar{u}_{\max }$ & $\bar{v}_{\max }$ & $\widetilde{U}$ & $\widetilde{V}$ & $\widetilde{Q}^{+}$ & $\widetilde{Q}^{-}$ \\
\hline \hline 25x100 & 185.5 & 151.6 & 19.5 & 34.7 & 3.67 & 3.67 \\
50x200 & 197.3 & 162.7 & 22.6 & 38.57 & 3.83 & 3.83 \\
$75 \times 300$ & 203.1 & 168.3 & 23.6 & 39.4 & 3.84 & 3.84 \\
100x400 & 206.02 & 169.1 & 23.9 & 39.7 & 3.85 & 3.85 \\
150x600 & 207.9 & 170.8 & 24.2 & 39.9 & 3.85 & 3.85 \\
\hline \hline
\end{tabular}

The evolution of all these quantities with the mesh density can be gathered from Table II, where it can clearly be seen that the percentage variation becomes smaller than $1 \%$ as soon as the resolution corresponding to the $75 \times 300$ (uniform) mesh is exceeded. Accordingly a mesh with 400 points along the $x$ axis and 100 points along the $y$ axis has been used for all the simulations.

A similar analysis has been carried out to test the particle tracking algorithm sensitivity to the mesh. Following Lappa (2018) this assessment has been based on the evaluation of "global” parameters defined as 


$$
\begin{gathered}
Q^{+}=\frac{\sum_{u_{p a r t}>0} m_{p a r t} u_{p a r t}}{M_{t o t}} \\
Q^{-}=\frac{\sum_{u_{p a r t}<0} m_{p a r t} u_{p a r t}}{M_{\text {tot }}} \\
\widetilde{Q}^{+}=\frac{\omega}{2 \pi} \int_{0}^{2 \pi / \omega} Q^{+} d t \\
\widetilde{Q}^{-}=\frac{\omega}{2 \pi} \int_{0}^{2 \pi / \omega} Q^{-} d t
\end{gathered}
$$

where $\mathrm{u}_{\text {part }}$ is the velocity component of each particle in the horizontal (x direction) and $\mathrm{m}_{\text {part }}$ is its mass (the parameter $Q^{ \pm} M_{\text {tot }}$ may therefore be seen as a measure of the overall particle instantaneous positive or negative horizontal momentum in the entire computational domain). The results for a representative set of parameters ( $\mathrm{Re}=1000, \omega=1000, \xi=2, \mathrm{St}=10^{-4}$ ), summarized in Fig. 4, demonstrate that the resolution corresponding to the $100 \times 400$ mesh is sufficient to make the particle dynamics mesh-independent as well (all curves collapse on a single curve as soon as the $75 \times 300$ resolution is exceeded; the same concept also applies to the corresponding time-averaged quantities defined by eq. (16), reported in Table II for the final cycle shown in Fig. 4).

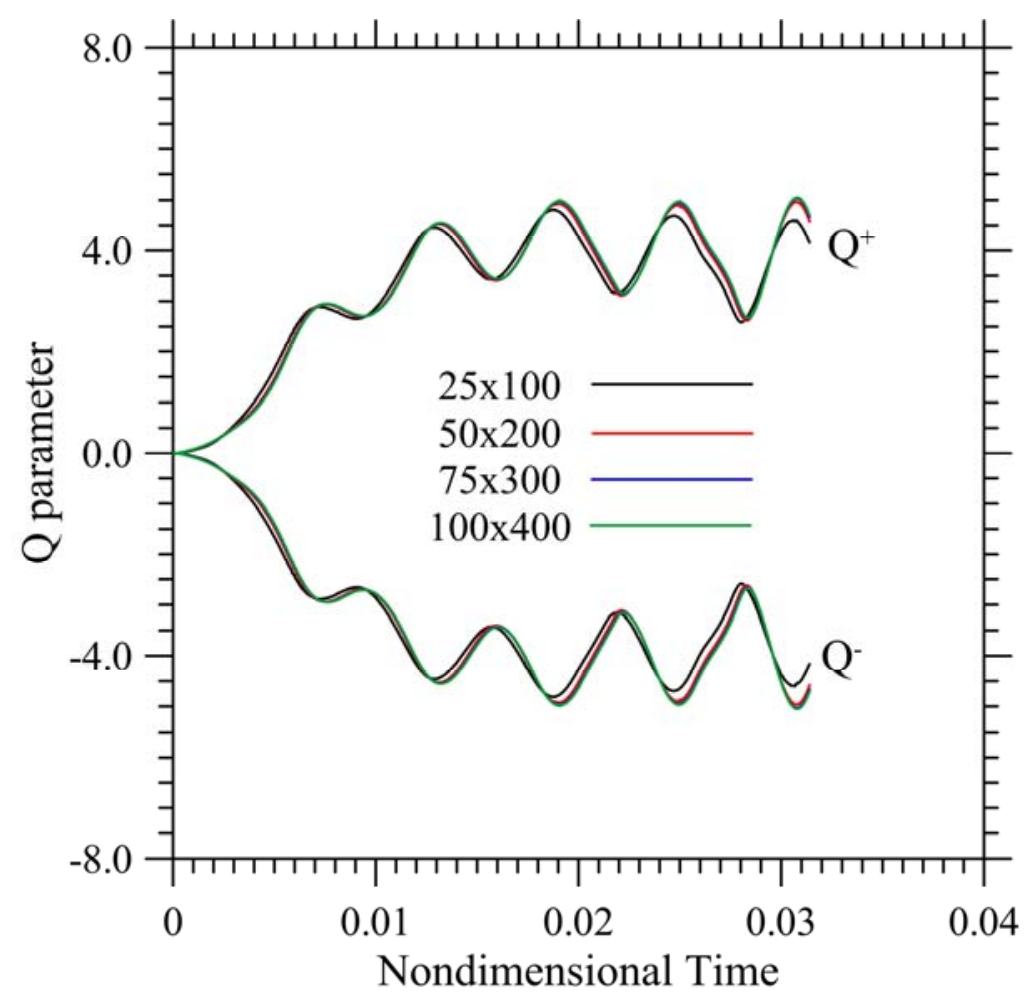

Figure 4: Parameters $Q^{+}$and $Q$ - versus time for different grids $(A=4, R e=1000, \omega=1000, \xi=2$, $\mathrm{St}=10^{-4}$, initial conditions corresponding to $\mathrm{N}_{\mathrm{r}} \mathrm{xN} \mathrm{N}_{\varphi}=5 \times 10^{3}$ spherical particles uniformly distributed over a circular region of non-dimensional radius $r=0.3$ ). 


\section{Results}

Stresses within unsteady simple shear flows of suspensions of inertial (non-Brownian) spheres constrained to move in the velocity-gradient plane are calculated using the model and numerical methods described in Sect. 2. The considered unsteady flows include oscillatory flows with shear reversal of varying strain amplitude and frequency as indicated at the end of Sect. 2.3. The evolution of the flow in time is reported along with the corresponding solid-mass micro-structural development for all flow conditions. It is a long-time process during which small deviations of the particle's trajectory from the streamlines of the instantaneous oscillatory flow accumulate until the behaviour becomes essentially non reversible. In the following we discuss a variety of results. All results are shown after a maximum of $\mathrm{M}=100$ cycles of modulation.

Given the theoretical background provided in the introduction and the stated intention of the present analysis to be a continuation (or an extension) of past studies on Stokesian dynamics to the case of $\operatorname{Re}>1$, it is convenient to start the description of the results from the case $R e=1$ (ideally separating the regime of Stokesian dynamics from the high-Re case).

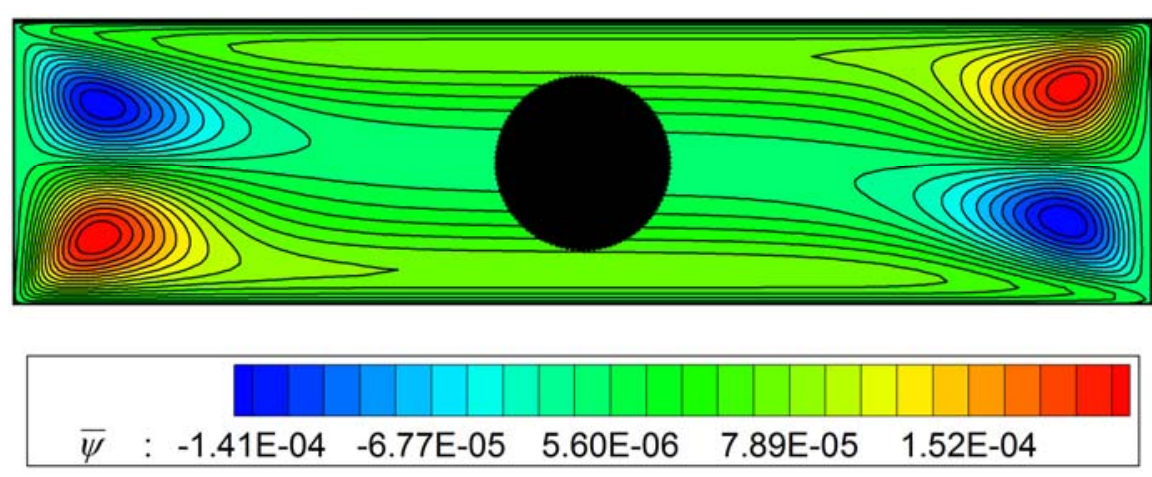

Figure 5: Snapshot of time-averaged velocity field and particle distribution for $\operatorname{Re}=1$ and $\omega=100$ after 100 cycles of modulation (apparently stationary dynamics).

Related numerical results are shown in Fig. 5 for a value of the forcing frequency $\omega=100$. It can be clearly seen that, as expected, in the absence of particle-to-particle interactions, the dynamics at low Reynolds number are fully reversible (the ball retains its initial perfect spherical shape at the end of any forcing cycle as time passes). Interestingly, the time-averaged flow for such conditions is very weak as witnessed by the very small values of the related streamfunction $\mathrm{O}\left(10^{-4}\right)$. Such flow consists essentially of four small vortices located in the corners of the cavity.

This case may be regarded as a trivial condition in which, when the fluid between the upper and lower walls is sheared by boundary motion that is subsequently reversed, then all fluid elements return almost perfectly to their starting positions.

However, the dynamics dramatically change when the Reynolds number is increased to $\operatorname{Re}=10$. The sequence of stages of evolution reported in Fig. 6 for $\omega=10$ clearly indicates that the shape of the set 
of particle (initially perfectly spherical) undergoes some distortion already after the first cycle of forcing (as evident in Fig. 6e).

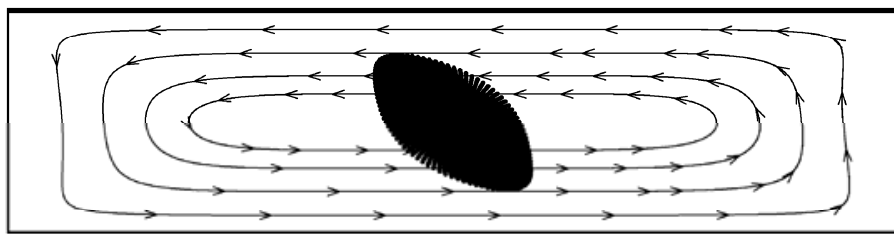

a)

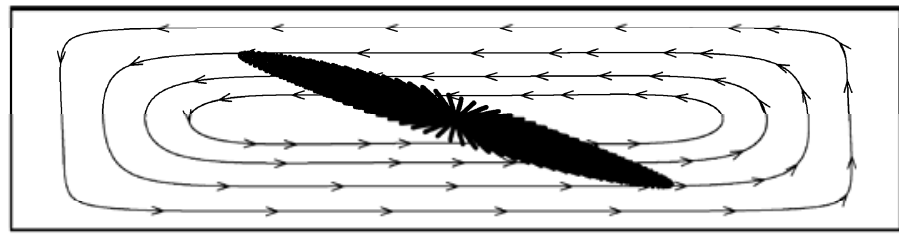

b)

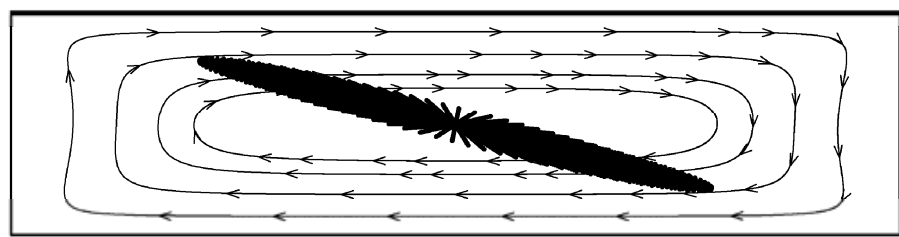

c)

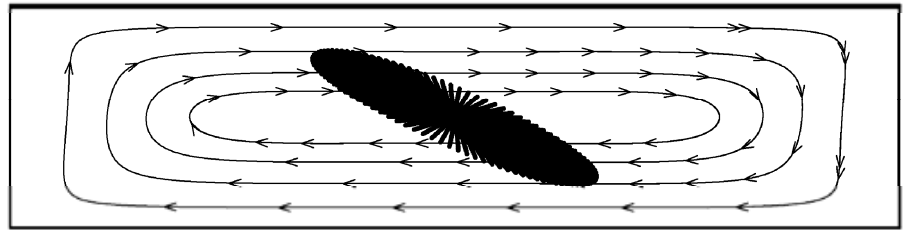

d)

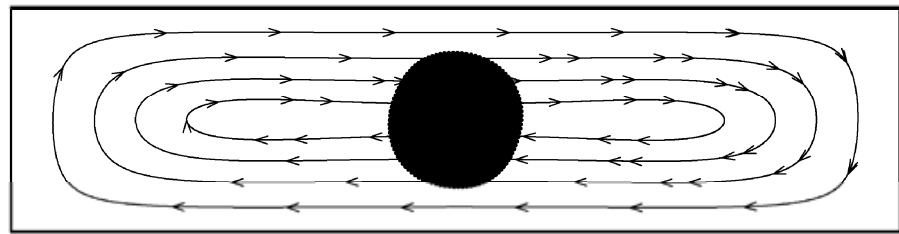

e)

Figure 6: Snapshots of velocity field and particle distribution as a function of time during the first cycle of modulation for $\mathrm{Re}=10$ and $\omega=10$ : a) $t=1 / 5 \tau_{\omega}$, b) $\left.\left.t=2 / 5 \tau_{\omega}, \mathrm{c}\right) \mathrm{t}=3 / 5 \tau_{\omega}, \mathrm{d}\right) \mathrm{t}=4 / 5 \tau_{\omega}$, e) $\mathrm{t}=\tau_{\omega}\left(\tau_{\omega}=2 \pi / \omega\right)$.

When repeating the cycles of shear, the deformation becomes even more evident and the cloud of particles progressively disperses in the fluid (Fig. 7). The set of particles, however, remains a cohesive entity for long times, maintaining a sharp boundary between its particle-filled interior and the clear fluid outside. In particular, its shape displays initially (after 10 cycles) the formation of two cusp points, which then develop into two well-defined and distinct branches as time further increases. After 100 cycles of modulation, the distribution of particles is finally stretched in a relatively long filament which extends from one edge to the other (Fig. 7e). 


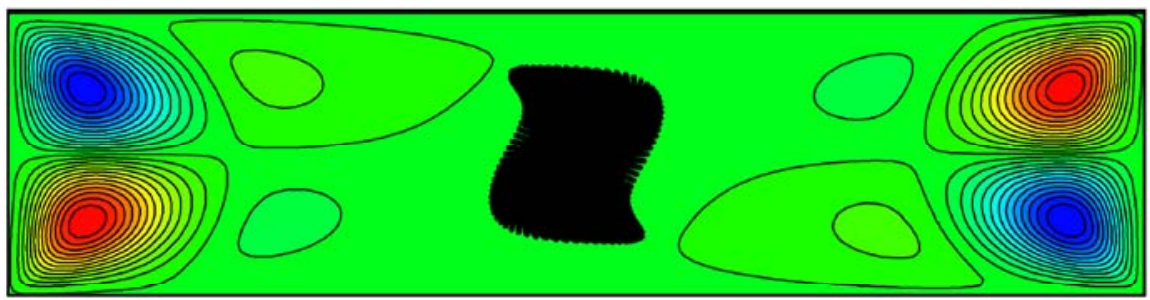

a)

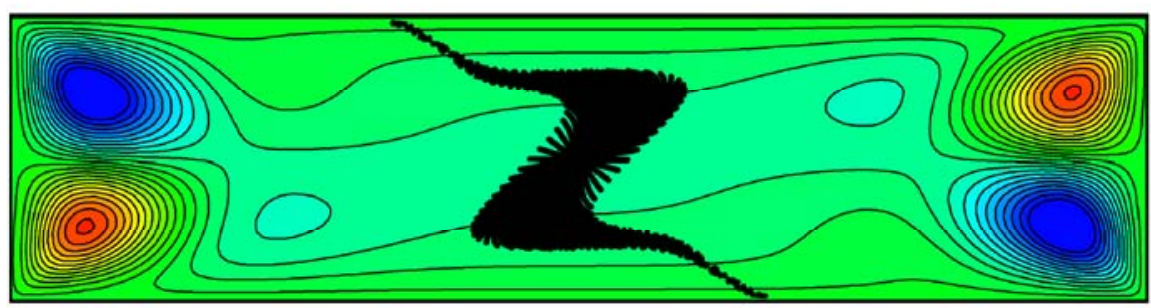

b)

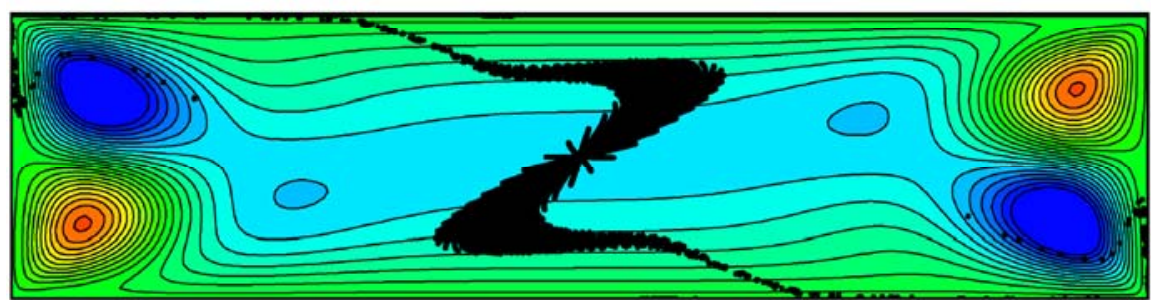

c)

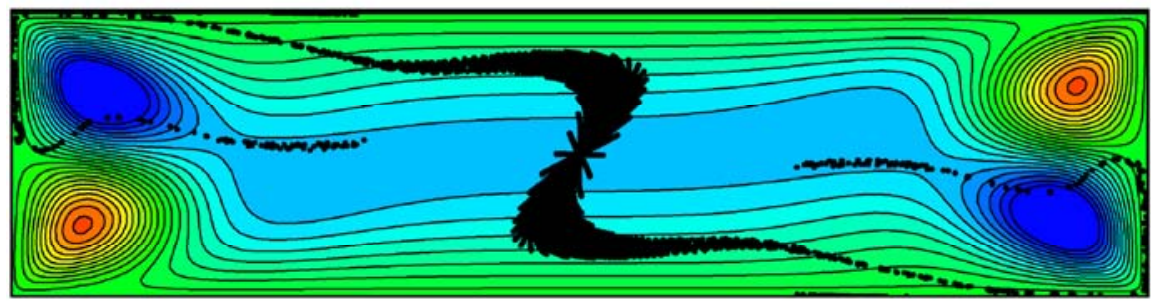

d)

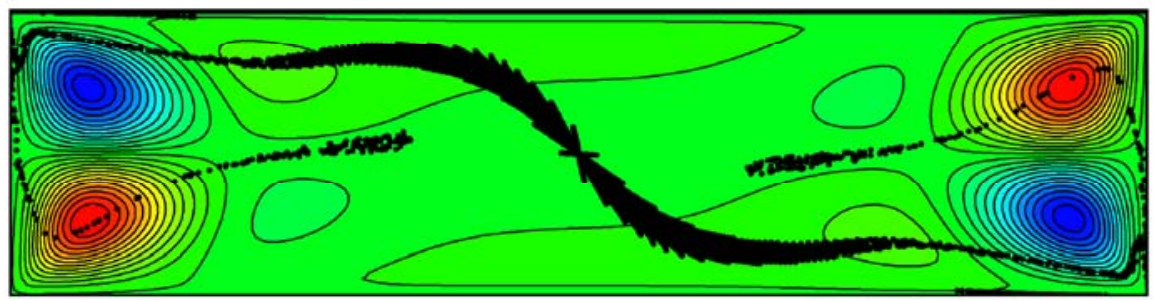

e)

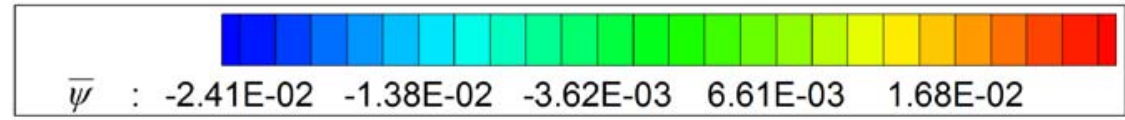

Figure 7: Snapshots of time-averaged velocity field and particle distribution as a function of time for $\operatorname{Re}=10$ and $\omega=10$ : a) $M=10$, b) 30 , c) 50 , d) 70 , e) 90 ( $M$ is the number of modulation cycles performed) (borderline dynamics). 

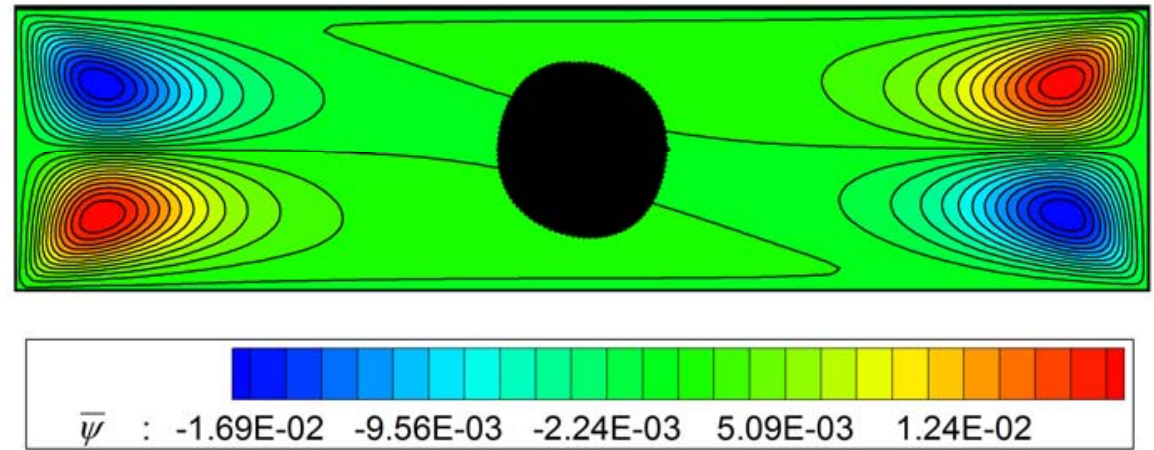

Figure 8: Snapshot of time-averaged velocity field and particle distribution for $\mathrm{Re}=10$ and $\omega=100$ after 100 cycles of modulation.

An increase (one order of magnitude) in the applied angular frequency while retaining the earlier value of the Reynolds number (i.e. $\operatorname{Re}=10, \omega=100$ ), however, determines a significant change; although the final shape taken by the set of particles after 100 cycles of modulation displays some deformation with respect to its initial spherical configuration, the oscillatorily sheared distribution of particles still exhibits a very compact ordering (Fig. 8).

This behaviour is retained if the angular frequency is further increased. Indeed for $\omega=1000$ the final shape of the ball is even more regular (Fig. 9).
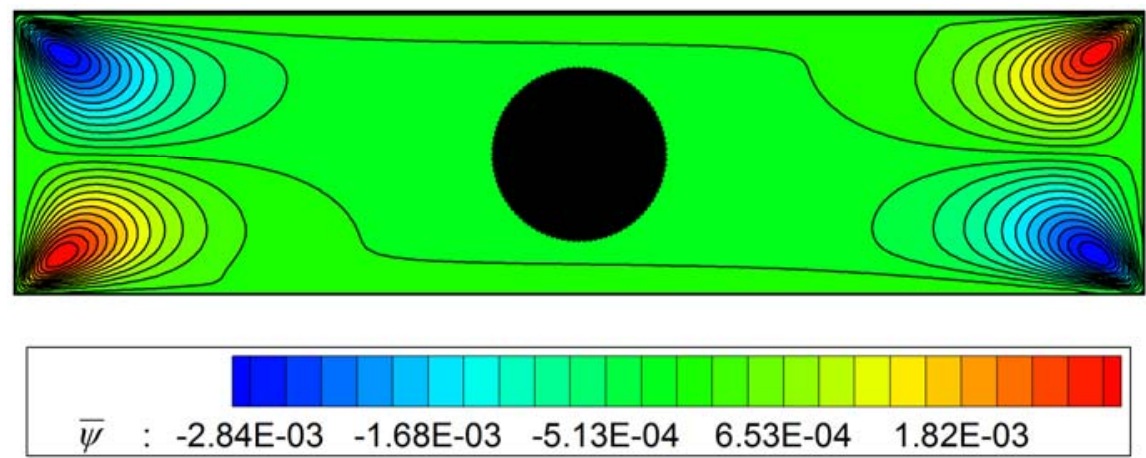

Figure 9: Snapshot of time-averaged velocity field and particle distribution for $\operatorname{Re}=10$ and $\omega=1000$ after 100 cycles of modulation (apparently stationary dynamics). 


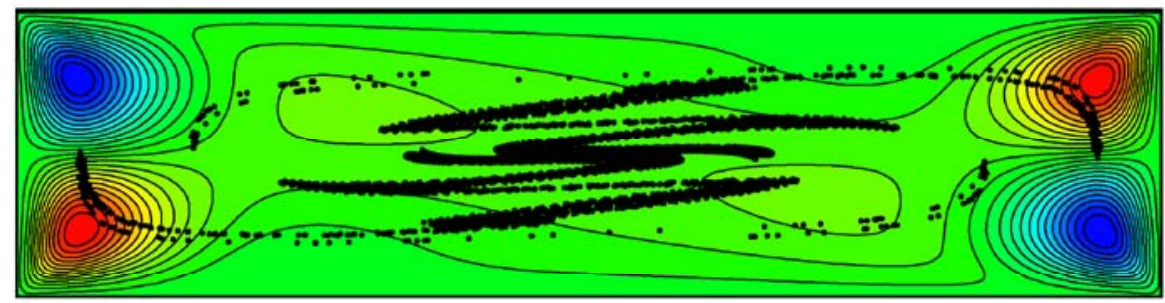

a)

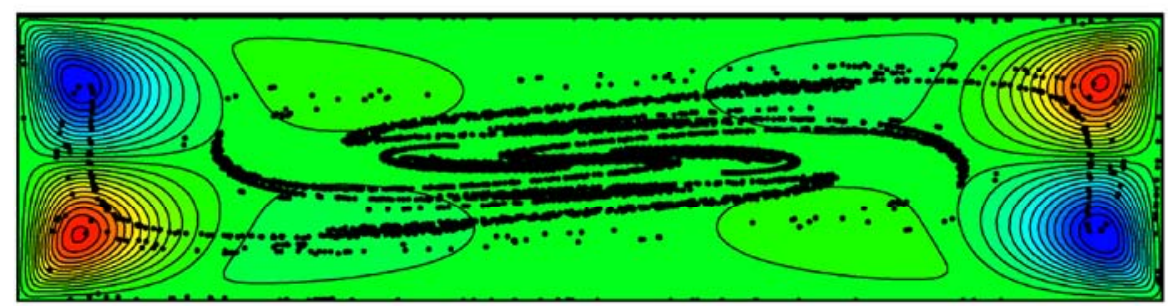

b)

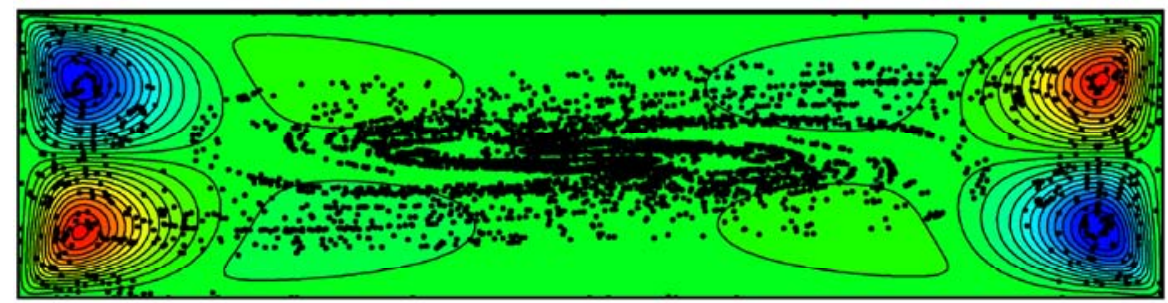

c)

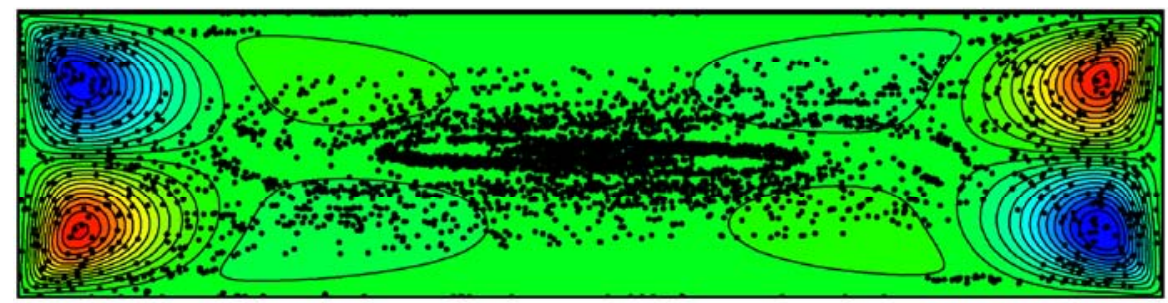

d)

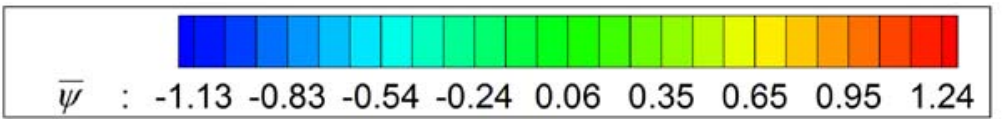

Figure 10: Snapshots of time-averaged velocity field and particle distribution as a function of time for $\operatorname{Re}=100$ and $\omega=10$ : a) $M=1$, b) 2, c) 5 , d) 10 ( $M$ is the number of modulation cycles performed) (chaotic dynamics with particle spiralling motion).

Figures 10-13 illustrate the dynamics for the same triplet of values of angular frequency considered for $\mathrm{Re}=10$ (covering three orders of magnitude, $\omega=10,100$ and 1000) when the Reynolds number is increased by one order of magnitude, i.e. $\mathrm{Re}=100$.

It can be seen that for this value of the Reynolds number, even a relatively small frequency is sufficient to prevent the compact ordering of particles. Indeed, by inspection of Figure 10, the reader will realise that already after a single cycle of forcing the original symmetry and cohesive behaviour of the set of particles are lost. Rather particles seem to order along some curved branches giving the illusion of spiralling filaments extended over the entire cavity. The set of particles does not remains a cohesive entity for long times and the identification of a sharp boundary between a 
particle-filled area and a clear fluid outside is no longer possible. Such structures seem to be uncorrelated from the underlying time-averaged velocity field shown on the background.

An increase in frequency (see Figs. 11 and 12 where $R e=100$ and $\omega=100$ ), however, leads to particle segregation phenomena similar to those already described for $\mathrm{Re}=10$ and $\omega=10$ (in particular, Fig. 11 shows the evolution over the first cycle of modulation, where it can be seen that for this value of frequency the system recovers some reversibility with respect to the dynamics seen for $\omega=10)$.
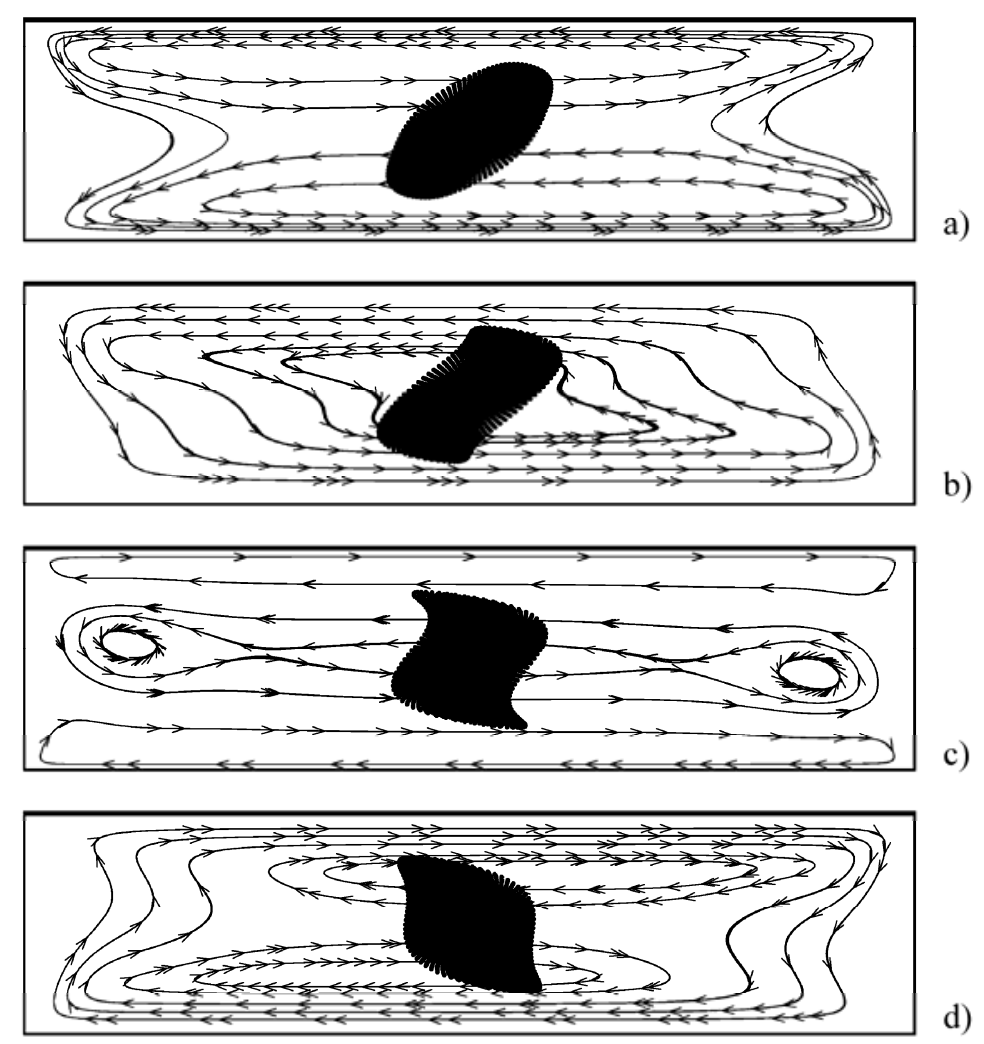

c)

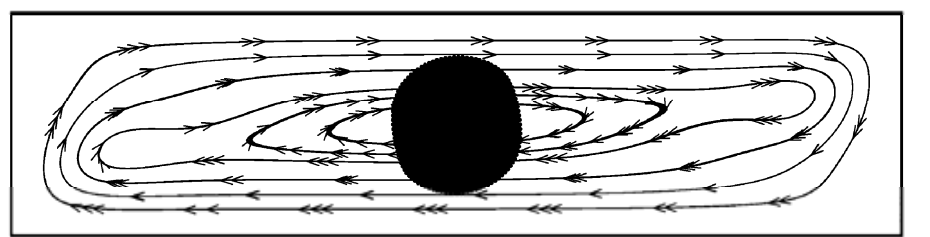

d)

e)

Figure 11: Snapshots of velocity field and particle distribution as a function of time during the first cycle of modulation for $\mathrm{Re}=100$ and $\omega=100$ : a) $t=1 / 5 \tau_{\omega}$, b) $t=2 / 5 \tau_{\omega}$, c) $t=3 / 5 \tau_{\omega}, d$ ) $t=4 / 5 \tau_{\omega}$, e) $\mathrm{t}=\tau_{\omega}\left(\tau_{\omega}=2 \pi / \omega\right)$.

Figure 12 shows the ensuing evolution as the number of performed cycles is increased.

The shape of the boundary separating the particle region from the external particle-free fluid develops some cusp points, which later evolve into extended branches. As a minor difference with respect to the dynamics observed in Fig. 7, however, the final structure attained by the particles after 100 cycles of forcing does not resemble that of an elongated S-shaped set. Rather particles 
tend to accumulate in proximity to the two moving boundaries while the core region of the cavity is progressively depleted as time passes.

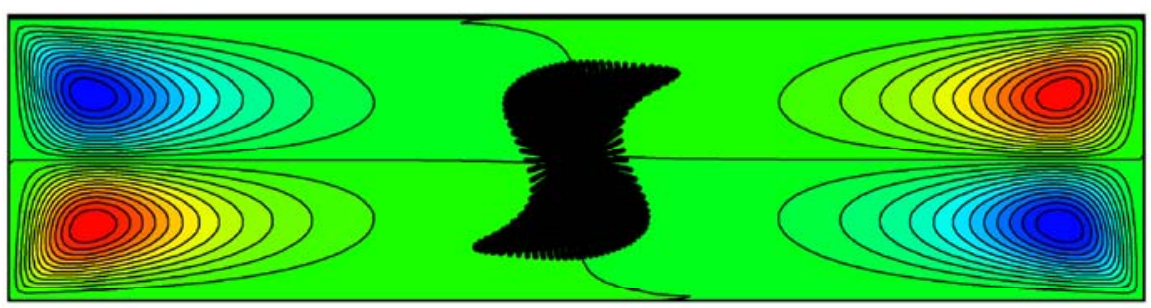

a)

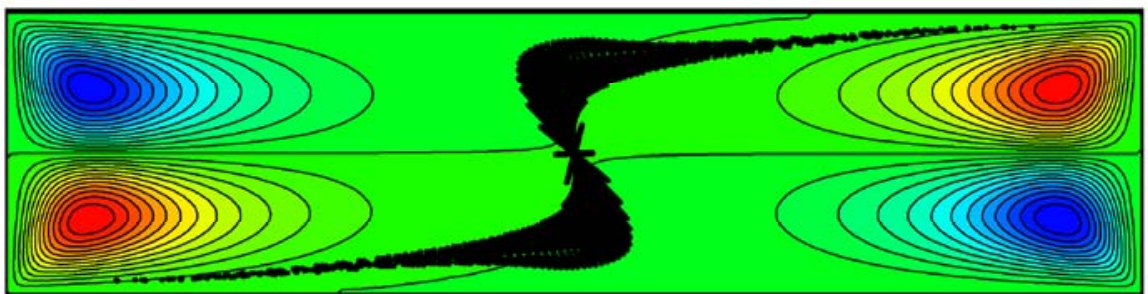

b)

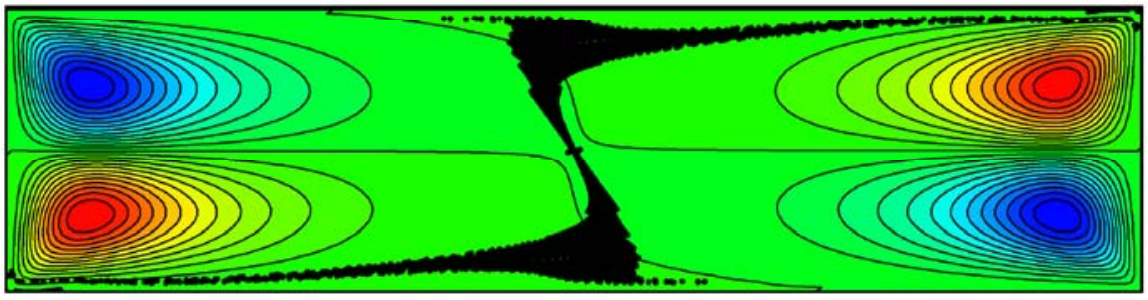

c)

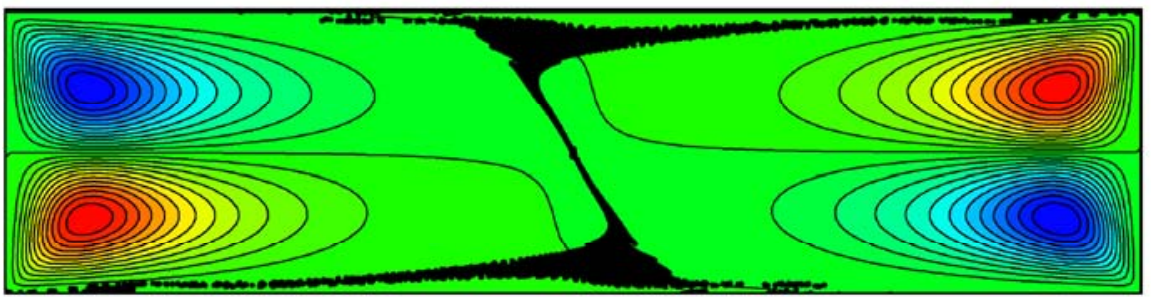

d)

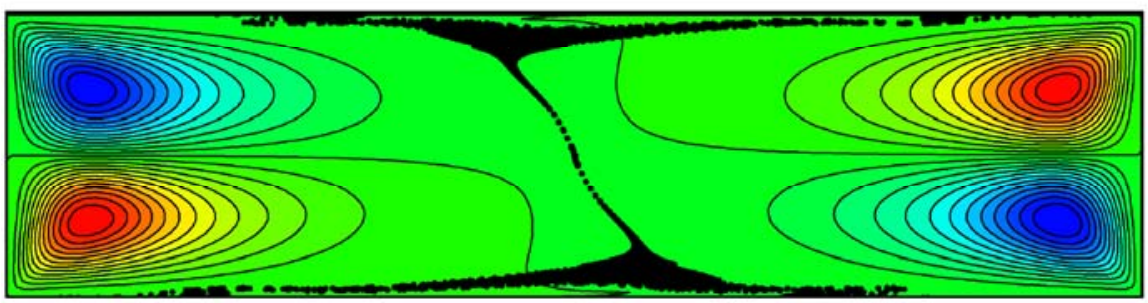

e)

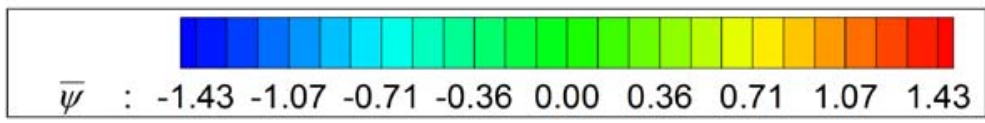

Figure 12: Snapshots of time-averaged velocity field and particle distribution as a function of time for $R e=100$ and $\omega=100$ : a) $M=10$, b) 30, c) 50, d) 70, e) 90 ( $M$ is the number of modulation cycles performed) - (borderline dynamics).

For this case some clear relationship can be discerned between the morphological and topological path taken by the set of particles during its evolution and the structure (pattern) of the underlying 
time-averaged velocity field shown on the background (the reader being also referred to the similar dynamics reported in Fig. 7).

Apparently, particles moving towards the corners 'feel' the presence of the vortices located there being captured by their streamlines and/or being subjected to the 'compressibility' effects described in the introduction (their trajectory departing from the path that would ideally be taken by mass-less and point tracers due to centrifugal effects).
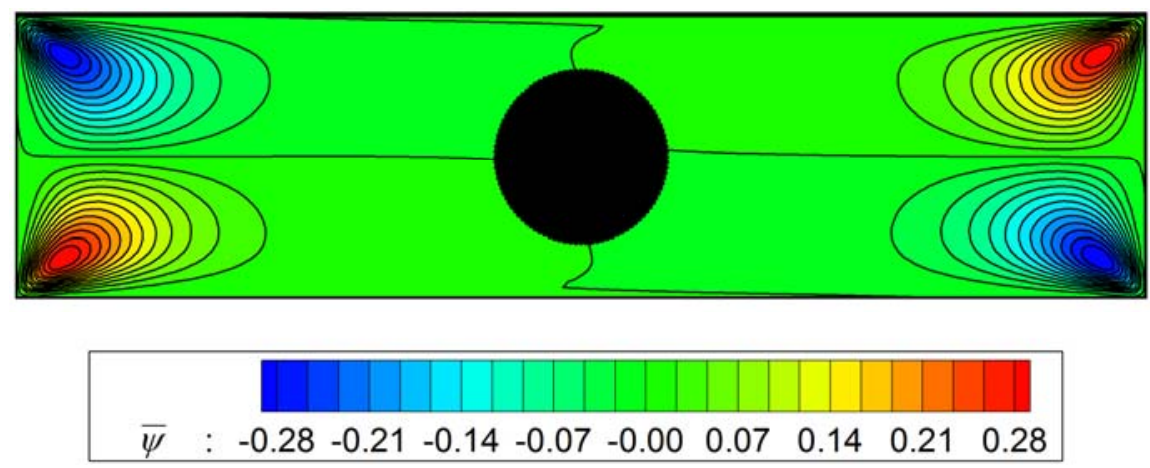

Figure 13: Snapshot of time-averaged velocity field and particle distribution for $\mathrm{Re}=100$ and $\omega=1000$ after 100 cycles of modulation (apparently stationary dynamics).

An increase in $\omega$ (to $\omega=1000$ ) leads to a recovery of reversible dynamics (Fig. 13).
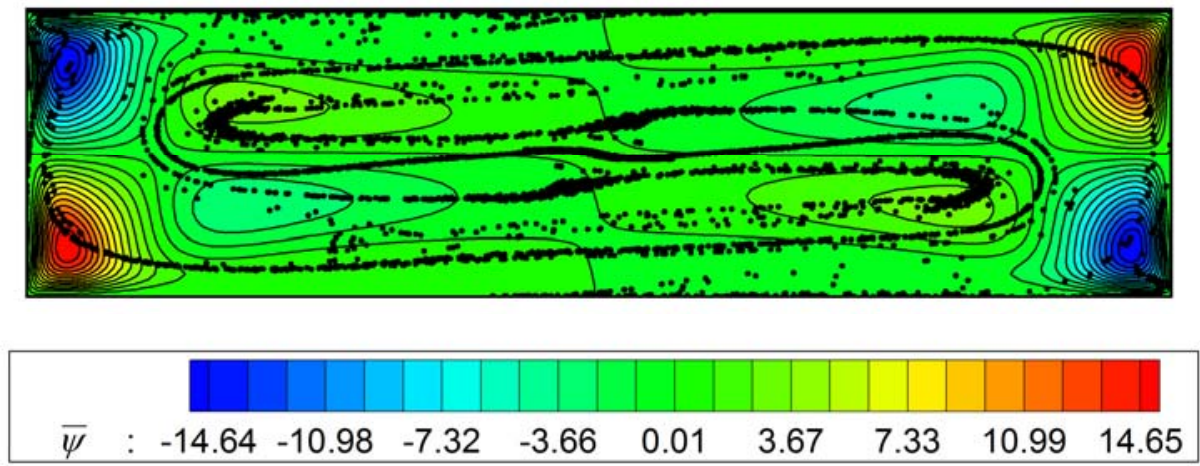

Figure 14: Snapshot of time-averaged velocity field and particle distribution for $\mathrm{Re}=1000$ and $\omega=100$ after 1 cycle of modulation (chaotic dynamics).

Figure 14 for $\operatorname{Re}=1000$ and $\omega=100$ shows again the same spiralling behaviours already discussed before for $\operatorname{Re}=100$ and $\omega=10$, which provides indirectly evidence for the existence of some recursion in the considered class of phenomena (the spiralling behaviour being apparently typical of situations where the order of magnitude of the angular frequency is smaller than that of the Reynolds number, i.e. $\mathrm{O}(\omega)<\mathrm{O}(\mathrm{Re}))$. 
When the angular frequency and the Reynolds number have again the same order of magnitude and $\mathrm{Re}=1000$ (Figs. 15), yet, as in the case $\mathrm{Re}=100$ and $\omega=100$ (Fig. 12), the numerical simulations seem to support the idea that in this case the time-averaged flow plays a crucial role in determining particle dynamics.

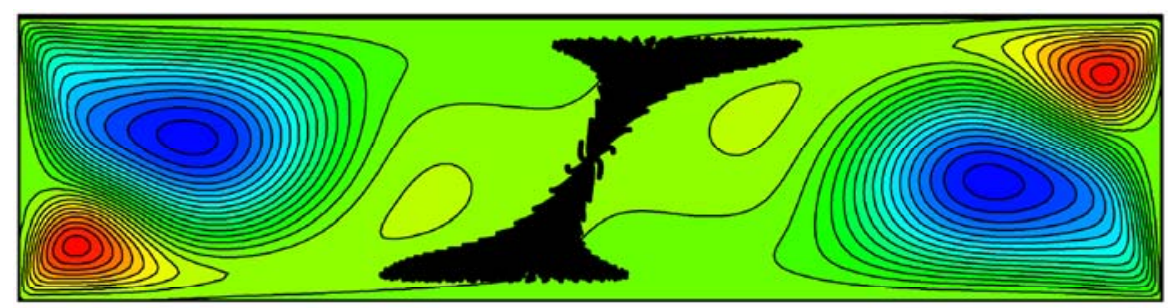

a)

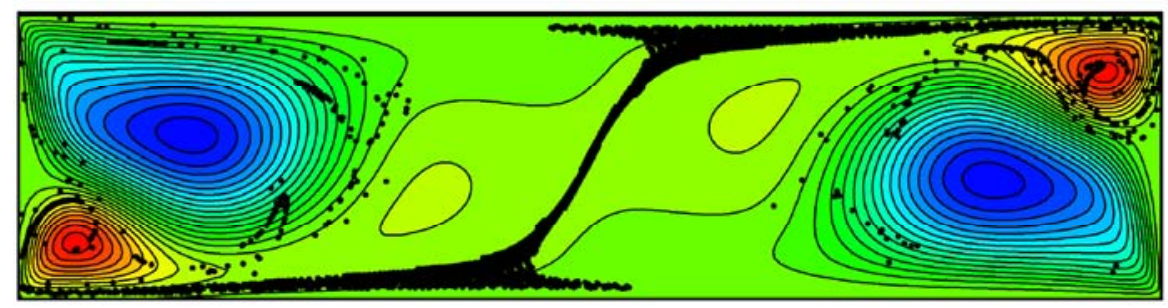

b)

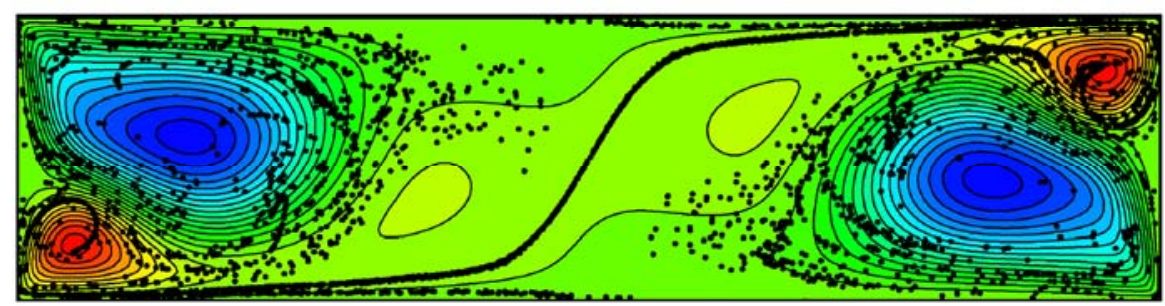

c)

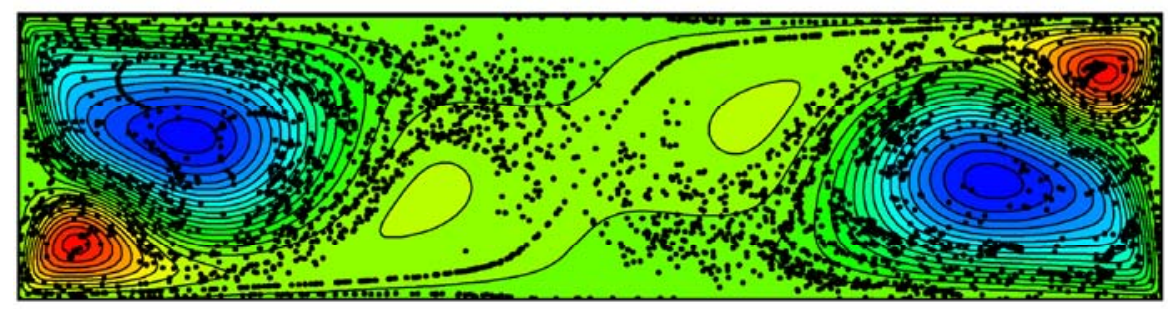

d)

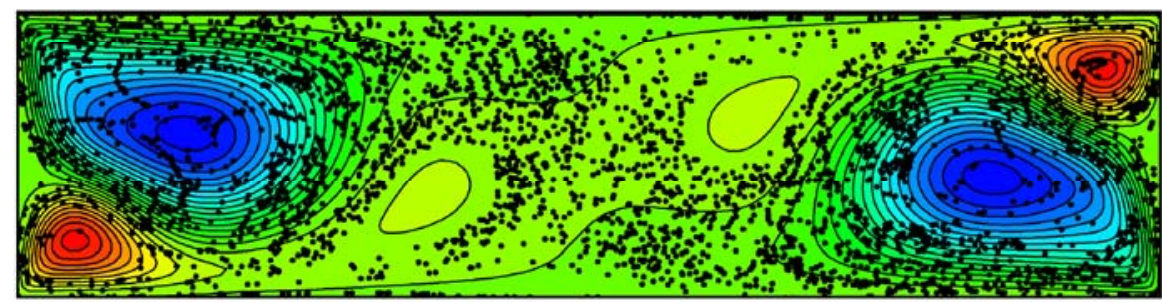

e)

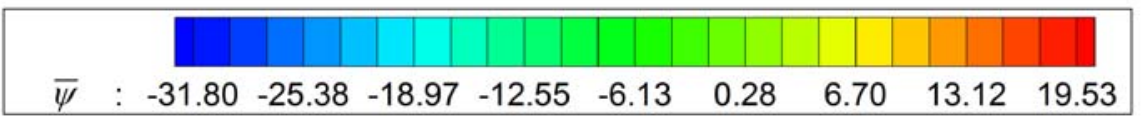

Figure 15: Snapshots of time-averaged velocity field and particle distribution as a function of time for $R e=1000$ and $\omega=1000$ : a) $m=10$, b) 20 , c) 30 , d) 40 , e) 50 ( $M$ is the number of modulation cycles performed) (irreversible dynamics). 
Indeed, Fig. 15 clearly proves that for such conditions the motion of particles is deeply influenced by the streamlines of the time-averaged flow. Interestingly, for $\operatorname{Re}=\omega=10^{3}$, the structure of the time-averaged flow is no longer symmetric like that seen for all preceding cases (with the four small vortices located in proximity to the corners). In this figure, indeed, the two vortices aligned along one of the two cavity diagonals (connecting opposite vertices of the rectangular domain) have size larger than that of the other two rolls.

Moreover, notably, the distribution of particles is chaotic. This result is not completely unexpected; in general, in fact, the dynamics of a small, but finite-size, spherical particle transported by a fluid flow obeying equations of motion that are inherently dissipative, can be chaotic even with a flow field that is simply time periodic (in the present case, as explained in Sect. 3.1, it is simply given by the sum of the steady time-averaged flow shown on the background of Fig. 15 and the instantaneous departures from such a steady flow experienced during each cycle of modulation by the fluid due to the applied forcing).

For $\operatorname{Re}=1000, \omega=10000$, finally the same behaviour already observed for $\operatorname{Re}=100$ and $\omega=1000$ is recovered (Fig. 16), which fully supports our earlier arguments about the fundamental role played by the ratio $\mathrm{Re} / \omega$ in determining the main properties of the system response to the application of forcing.
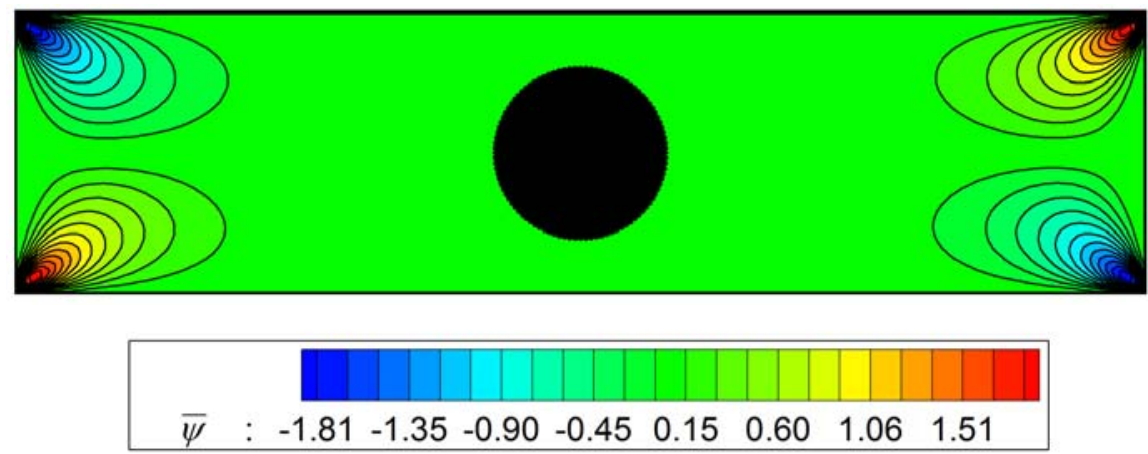

Figure 16: Snapshot of time-averaged velocity field and particle distribution for $\mathrm{Re}=1000$ and $\omega=10000$ after 100 cycles of modulation (apparently stationary dynamics; for this specific case a mesh 300x1200 has been used to obtain a grid-independent solution).

\section{Discussion \& Conclusions}

It needs no demonstration that if the cloud of particles does not reconstitute identically at the end of each cycle of shear, but instead progressively expands and/or undergoes some change in shape, a contribution to the particle velocity with non-zero average value in time must enter the dynamics somewhere. Indeed, these concepts are known since the original works by Herringe (1977) and Ikeda (1989), who focused on the settling or rising behaviours of a single spherical particle in an oscillating fluid (by imposing combined vertical and/or horizontal oscillations on a liquid, these authors could control particle mean motion allowing particles either to fall with a decreased velocity or even to rise against gravity). 
In the present study, however, we have shown (see Figs. 5-16) that the extent of irreversibility in the particle dynamics strongly correlates with the ratio of the Reynolds number to the non-dimensional frequency.

In the following, we will show how, in order to understand the origin of such a correlation and improve our knowledge of the related underlying cause-and-effect relationships, it is convenient to concentrate on the typical characteristic lengths involved in the considered phenomena, these being:

1) The space that the generic particle located on the initial ball has to travel in order to reach the regions where time-averaged convection is significant and can therefore have an impact on its velocity;

2) The space effectively covered by a particle during half of an oscillation period (before the velocity of the lid changes sign and the particle tends to be moved back towards its initial location).

Because, as we have seen in Figs. 5, 7-10 and 12-16, rolls of steady time-averaged convection are located in proximity to the lateral walls, the former characteristic length may be assumed to be approximately half of the system extension along the horizontal direction, i.e. $\ell / 2$.

Given its definition, the latter characteristic (dimensional) length will obviously depend on the amplitude and frequency of velocity at the two moving boundaries. We may assume it to be given by the time integral:

$$
\int_{0}^{\pi / \Omega} \Lambda \sin (\Omega t) d t=-\frac{\Lambda}{\Omega}[\cos (\Omega t)]_{0}^{\pi / \Omega}=\frac{2 \Lambda}{\Omega}
$$

Therefore, the condition by which a particle subjected to oscillatory shear can effectively reach one of the two extremities of the cavities and be influenced there by strong steady convection (particles being captured directly by the corner vortices and/or being subjected there to the related centrifugal effects) can be expressed as:

$$
\frac{\ell}{2}=\frac{2 \Lambda}{\Omega}
$$

which, recalling the non-dimensional quantities defined in Sect 2. can be also recast in condensed form as:

$$
\frac{\mathrm{Re}}{\omega}=\frac{A}{4}
$$


where $\mathrm{A}$ is the aspect ratio ( $\mathrm{A}=4$ in our case). From this relationship it can be argued that for $\mathrm{A}=4 \mathrm{a}$ generic particle will reach the lateral regions if $\operatorname{Re}>\omega$, the condition $\operatorname{Re}=\omega$ representing a limit (transition) situation. By providing at the same time an excellent correlation of numerical results on the one hand, and a relatively simple physical interpretation of observed behaviour on the other hand, equation (19) may be regarded as an additional limit condition to be considered for separating reversible and irreversible dynamics in the space of parameters.

On the basis of the present study, the following conclusions can therefore be drawn:

- A minimal model was developed to inquire specifically about the role of non-linear convective effects in determining the departure from reversible and deterministic dynamics of a system of solid particles suspended in a fluid and subjected to periodic shear reversal.

- To isolate this problem with respect to earlier studies and theories available in the limit as dissipation dominates and inertia can be ignored, we have not considered inter-particle forces.

- We have found that three conditions combine essentially in determining the system dynamics and its apparently deterministic or chaotic behaviour over a given time horizon:

1. The set of particles can be expected to deform while remaining a cohesive entity over the considered initial timeframe if the time-averaged displacement of the generic particle over the considered time is orders of magnitude smaller than the instantaneous displacements (which, as witnessed by the small values of $\bar{\psi}_{\max }$ reported in the figure captions generally happens for $\mathrm{O}(\omega) \geq \mathrm{O}(\mathrm{Re}))$.

2. By contrast, a quick dispersion of particles (spiralling behaviour) is obtained when the timeaveraged and instantaneous displacements of the generic particle have a comparable order of magnitude (relatively high $\mathrm{Re}$ and smaller $\omega$, this condition destroying almost immediately the initially ordered configuration, i.e. the ball).

3. Furthermore, a precise threshold exists for the ratio of the velocity amplitude to frequency beyond which particles do not return to their starting configurations after one or more cycles. Instead, their displacements are influenced by the streamlines of the time-averaged fluid flow established in the cavity as a consequence of the non-linear nature of the governing Navier-Stokes equations and finite size of the fluid container.

4. At relatively high values of Re and $\omega$, instability phenomena affecting the structure and intensity of the time-averaged flow can determine transition to chaotic behaviour even for situations in which $\mathrm{O}(\omega)=\mathrm{O}(\mathrm{Re})\left(\mathrm{Re}=\omega=10^{3}\right.$ in the present simulations).

Future work shall be devoted to test the above principles in different conditions and expand the considered space of parameters by examining different values or ranges of the particle Stokes number and density ratio (including the case in which the particles have density smaller than the 
external fluid). Moreover, companion studies may take into account the effect of gravity (expected to break the system symmetry with respect to the direction perpendicular to the moving walls, thereby producing significant alterations in the particle patterning behaviour).

\section{Acknowledgments}

This work has been partially supported by the UK Space Agency (grant ST/S006354/1) in the framework of the T-PAOLA project.

\section{References}

[-] Atkinson K.A., An Introduction to Numerical Analysis (2nd ed.), (John Wiley \& Sons, New York, 1989).

[-] Baird M. H. I., Senior M. G., and Thompson R. J., (1967), Terminal Velocities of Spherical Particles in a Vertically Oscillating Liquid, Chem. Eng. Sci., 22, 551-558.

[-] Benczik I. J., Toroczkai Z., and Tél T., (2002), Selective Sensitivity of Open Chaotic Flows on Inertial Tracer Advection: Catching Particles with a Stick, Phys. Rev. Lett., 89 (16), 164501

[-] Brown C.J, McGlone T., Yerdelen S. and other 24 authors, (2018), Enabling precision manufacturing of active pharmaceutical ingredients: workflow for seeded cooling continuous crystallisations, Molecular Systems Design \& Engineering, DOI: 10.1039/c7me00096k

[-] Carotenuto L., Piccolo C., Castagnolo D., Lappa M., Garcìa-Ruiz J.M., (2002), Experimental observations and numerical modelling of diffusion-driven crystallisation processes, Acta Crystallographica D, 58: 1628-1632.

[-] Chen L., Goto S. and Vassilicos J. C., (2006), Turbulent clustering of stagnation points and inertial particles, J. Fluid Mech., 553, 143-154.

[-] Coimbra F.M. and Rangel R.H. (2001), Spherical particle motion in harmonic stokes flows, AIAA J, 39(9), 1673-1682.

[-] Corté L., Chaikin P. M., Gollub J. P. and Pine D. J., (2008), Random organization in periodically driven systems, Nature Physics 4, 420-424.

[-] Deng X. and Chawla N., (2006), Modeling the effect of particle clustering on the mechanical behaviour of SiC particle reinforced Al matrix composites, Journal of Materials Science, 41(17), 5731-5734.

[-] Eaton J.K. and Fessler J.R., (1994), Preferential concentration of particles by turbulence, Int. J. Multiphase Flow, 20, 169-209.

[-] Gotoda M., Melnikov D. E., Ueno I., and Shevtsova V., (2016), Experimental study on dynamics of coherent structures formed by inertial solid, particles in three-dimensional periodic flows, Chaos, 26, 073106 (11 pages).

[-] Haller G. and Sapsis T., (2008), Where do inertial particles go in fluid flows?, Physica D: Nonlinear Phenomena, 237(5), 573-583.

[-] Herringe R.A., (1977), A study of particle motion induced by two-dimensional liquid oscillations, Int. J. Multiphase Flow, 3(3), 243-253

[-] Hjelmfelt A. T., and Mockros L. F., (1966), Motion of Discrete Particles in a Turbulent Fluid, Appl. Sci. Res., 16(1), 149-161.

[-] Houghton G., (1961), The behaviour of particles in a sinusoidal vector field, Proc. Roy. Soc. A 272, 33-43.

[-] Ikeda S., (1989), Fall Velocity of Single Spheres in Vertically Oscillating Fluids, Fluid Dyn. Res., 5, 203-216.

[-] Ilin K. and Morgulis A., (2012), On the steady streaming induced by vibrating walls, SIAM Journal on Applied Mathematics, 72(5): 1406-1427. 
[-] Ilin K. and Morgulis A., (2015), Steady streaming in a vibrating container at high Reynolds numbers, Proceedings of XLIII ADVANCED PROBLEMS IN MECHANICS 2015 International Conference

[-] Ivanova, A.A., Kozlov, V.G. and Kuzaev, A.F., (2005), Vibrational lift force acting on a body in a fluid near a solid surface. Dokladi RAN 2005, 402, 1-4 (Translated. Doklady Physics, 50, 311314).

[-] Karmakar H. and Pandit S.K., (2015), Numerical Solutions of Incompressible Viscous Flows in a Double-Lid-Driven Cavity, Chapter 23 in S. Sarkar et al. (eds.), Applied Mathematics, Springer Proceedingsin Mathematics \& Statistics 146, DOI 10.1007/978-81-322-2547-8_23

[-] Kozlov, V.G., Ivanova, A.A. and Evesque, P., (2006), Block stratification of sedimenting granular matter in a vessel due to vertical vibration, Fluid Dyn. Mater. Process., 2, 203-210.

[-] Kuhlmann H.C., Lappa M., Melnikov D., Mukin R., Muldoon F.H., Pushkin D., Shevtsova V.S., and Ueno I., (2014), The JEREMI-Project on thermocapillary convection in liquid bridges. Part A: Overview of Particle Accumulation Structures, Fluid Dyn. Mater. Process., 10 (1): 1-36.

[-] Ladyzhenskaya O.A., (1969), The Mathematical Theory of Viscous Incompressible Flow, Gordon and Breach, 2nd Edition, New York - London, 1969.

[-] Langbein D., (1991), Motion of Ensembles of Spherical Particles in a Fluid Due to g-jitter, Adv. Space Res., 11(7), 189-196.

[-] Lappa M. and Carotenuto L., (2003), Effect of convective disturbances induced by g-jitter on the periodic precipitation of lysozyme, Microgravity Science \& Technology, XIV/2, 41-56.

[-] Lappa M., (1997), Strategies for parallelizing the three-dimensional Navier-Stokes equations on the Cray T3E; Science and Supercomputing at CINECA, 11, 326-340. ISBN-10: 88-86037-03-1, M. Voli Editor, Bologna, 1997.

[-] Lappa M., (2005) Assessment of VOF Strategies for the analysis of Marangoni Migration, Collisional Coagulation of Droplets and Thermal wake effects in Metal Alloys under Microgravity conditions, Computers, Materials \& Continua CMC, 2(1), 51-64.

[-] Lappa M., (2013a), Assessment of the role of axial vorticity in the formation of Particle Accumulation Structures (PAS) in supercritical Marangoni and hybrid thermocapillary-rotationdriven flows, Physics of Fluids, 25(1) 012101 (11 pages).

[-] Lappa M., (2013b), On the Existence and Multiplicity of One-dimensional Solid Particle Attractors in Time-dependent Rayleigh-Bénard Convection, Chaos, 23(1), 013105 (9 pages).

[-] Lappa M., (2013c), On the variety of particle accumulation structures under the effect of gjitters, Journal of Fluid Mechanics, 726: 160-195.

[-] Lappa M., (2014a), Stationary Solid Particle Attractors in Standing Waves, Physics of Fluids, 26(1), 013305 (12 pages).

[-] Lappa M., (2014b), The patterning behaviour and accumulation of spherical particles in a vibrated non-isothermal liquid, Physics of Fluid, 26(9), 093301 (22 pages).

[-] Lappa M., (2016a), Numerical study into the morphology and formation mechanisms of threedimensional particle structures in vibrated cylindrical cavities with various heating conditions, Physical Review Fluids, 1(6), 064203 (25 pages).

[-] Lappa M., (2016b), On the nature, formation and diversity of particulate coherent structures in Microgravity Conditions and their relevance to materials science and problems of Astrophysical interest, Geophysical and Astrophysical Fluid Dynamics, 110(4): 348-386.

[-] Lappa M., (2016c), Control of convection patterning and intensity in shallow cavities by harmonic vibrations, Microgravity Science \& Technology, 28(1), 29-39.

[-] Lappa M., (2017), On the multiplicity and symmetry of particle attractors in confined nonisothermal fluids subjected to inclined vibrations, Int. J. Multiphase Flow, 93: 71-83.

[-] Lappa M., (2018), On the transport, segregation and dispersion of heavy and light particles interacting with rising thermal plumes, Physics of Fluids, 30(3), 033302 (23 pages).

[-] Lappa M., Piccolo C., Carotenuto L., (2003), Numerical and experimental analysis of periodic patterns and sedimentation of lysozyme, J. Cryst. Growth, 254/3-4: 469-486. 
[-] Lee H.M. and Park O.O., (1994), Rheology and dynamics of immiscible polymer blends, Journal of Rheology, 38, 1405-1425.

[-] Lighthill M. J., (1954), The Response of Laminar Skin Friction and Heat Transfer to Fluctuations in the Stream Velocity, Proc. R. Soc. London, Ser. A, 224, 1-23.

[-] Markov A. V., (2008), Thermal conductivity of polymers filled with dispersed particles: A model, Polymer Science Series A, 50(4), 471-479.

[-] Maxey M. R. and Riley J. J., (1983), Equation of motion for a small rigid sphere in a nonuniform flow, Phys. Fluids, 26, 883-889.

[-] Mei R., Lawrence J., and Adrian J., (1991), Unsteady Drag on a Sphere at Finite Reynolds Number With Small Fluctuations in the Free-Stream Velocity, J. Fluid Mech., 233, 613-631

[-] Melnikov D., Pushkin D., and Shevtsova V., (2011), Accumulation of particles in timedependent thermocapillary flow in a liquid bridge. Modeling of experiments, Eur. Phys. J. Special Topics 192, 29.

[-] Metzger B. and Butler J. E., (2010), Irreversibility and chaos: Role of long-range hydrodynamic interactions in sheared suspensions, Phys. Rev. E, 82, 051406

[-] Metzger B. and Butler J. E., (2012), Clouds of particles in a periodic shear flow, Phys. Fluids., 24, 021703 (6 pages)

[-] Michaelides E.E., (1997), Review - The transient equation of motion for particles, bubbles, and droplets, J. Fluids Eng., 119, 233-247.

[-] Perumal D.A. and Dass A. K., (2008), Simulation of flow in two-sided lid-driven square cavities by the lattice Boltzmann method, Advances in Fluid Mechanics VII, WIT Transactions on Engineering Sciences (2008, WIT Press), Vol 59, pp. 45-54. doi:10.2495/AFM080051

[-] Pine D. J., Gollub J. P., Brady J. F., and Leshansky A. M., (2005), Chaos and threshold for irreversibility in sheared suspensions, Nature (London) 438, 997-1000.

[-] Prandtl L., (1938), "Zur berechnung der grenzschichten", ZAMM-Journal of Applied Mathematics and Mechanics/Zeitschrift für Angewandte Mathematik und Mechanik 18.1: 77-82.

[-] Pushkin D., Melnikov D., Shevtsova V., (2011), Ordering of Small Particles in OneDimensional Coherent Structures by Time-Periodic Flows, Phys. Rev. Lett., 106, 234501.

[-] Raju N. and Meiburg E., (1995), The accumulation and dispersion of heavy particles in forced two-dimensional mixing layers. Part 2: The effect of gravity, Phys. Fluids, 7, 1241-1264.

[-] Ratke L., Korekt G., Drees S., (1996), Solidification of immiscible alloys, ESA SP 385, pag. 247.

[-] Sapsis T. and Haller G., (2010), Clustering criterion for inertial particles in two-dimensional time-periodic and three-dimensional steady flows, Chaos, 20, 017515.

[-] Savino R. and Lappa M., (2003), “Assessment of the thermovibrational theory: application to gjitter on the Space-station”, Journal of Spacecraft and Rockets, 40 (2): 201-210.

[-] Schwabe D. and Mizev A. I., (2011), Particles of different density in thermocapillary liquid bridges under the action of travelling and standing hydrothermal waves, Eur. Phys. J. Spec. Top. 192, 13-27.

[-] Schwabe D., Mizev A.I., Udhayasankar M. and Tanaka S., (2007), Formation of dynamic particle accumulation structures in oscillatory thermocapillary flow in liquid bridges, Phys. Fluids, 19(7), 072102.

[-] Schwabe, D., Hintz, P. and Frank, S., (1996), New features of thermocapillary convection in floating zones revealed by tracer particle accumulation structures, Microgravity Sci. Tech., 9, 163168.

[-] Shevtsova V., Melnikov D., Ryzhkov I., Gaponenko Y., (2010), Experimental and theoretical study of vibration-induced thermal convection in low gravity, J. Fluid Mech., 648, 53-82.

[-] Shevtsova V., Mialdun A., Melnikov D., Ryzhkov I., Gaponenko Y., Saghir Z., Lyubimova T., Legros J.C., (2011), The IVIDIL experiment onboard the ISS: Thermodiffusion in the presence of controlled vibrations, Comptes Rendus Mécanique, 339 (5), 310-317. 
[37] Shevtsova V., Lyubimova T., Saghir Z., Melnikov D., Gaponenko Y., Sechenyh V., Legros J.C. and Mialdun A., (2011), IVIDIL: on-board g-jitters and diffusion controlled phenomena, J. Phys.: Conf. Ser., 327, 012031.

[-] Simic-Stefani S., Kawaji M. and Hu H., (2006), G-jitter induced motion of a protein crystal under microgravity, J. Cryst. Growth, 294, 373-384.

[-] Tabakova S.S. and Zapruanov Z.D., (1982a), On the hydrodynamic interaction of two spheres oscillating in a viscous fluid.I. Axisymmetrical case, J. Appl. Math. Phys. 33, 344-357; (1982b), On the hydrodynamic interaction of two spheres oscillating in a viscous fluid. II. Three dimensional case, ibid. 33, 487-502.

[-] Thomson J.R., Casademunt J., Drolet F. and Vinals J., (1997), Coarsening of solid-liquid mixtures in a random acceleration field, Phys Fluids, 9(5), 1336-1343.

[-] Tunstall E. B., and Houghton G., (1968), Retardation of Falling Spheres by Hydrodynamic Oscillations, Chem. Eng. Sci., 23, 1067-1081.

[-] Vojir D. J. and Michaelides E. E., (1994), The Effect of the History Term on the Motion of Rigid Spheres in a Viscous Fluid, Int. J. Multiphase Flow, 20, 547-556.

[-] Voth G. A., Bigger B., Buckley M.R., Losert W., Brenner M.P., Stone H.A., and Gollub J.P., (2002), Ordered clusters and dynamical states of particles in a vibrated fluid. Phys. Rev. Lett. 88, 234301.

[-] Wunenburger R., Carrier V., and Garrabos Y., (2002), Periodic order induced by horizontal vibrations in a two-dimensional assembly of heavy beads in water, Phys. Fluids 14, 2350.

[-] Zhang Q., Li W., Gu M. and Yanping J., (2006), Dispersion and rheological properties of concentrated silicon aqueous suspension, Powder Technology,161,130-134

[-] Zhao J. Z., Ratke L. and Feuerbacher B., (1998), Microstructure evolution of immiscible alloys during cooling through the miscibility gap, Modelling Simul. Mater. Sci. Eng., 6, 123-139.

[-] Zhao J.Z., Ahmed T., Jiang H.X., He J., Sun Q., (2017), Solidification of Immiscible Alloys: A Review, Acta Metallurgica Sinica (English Letters), 30(1), 1-28. 